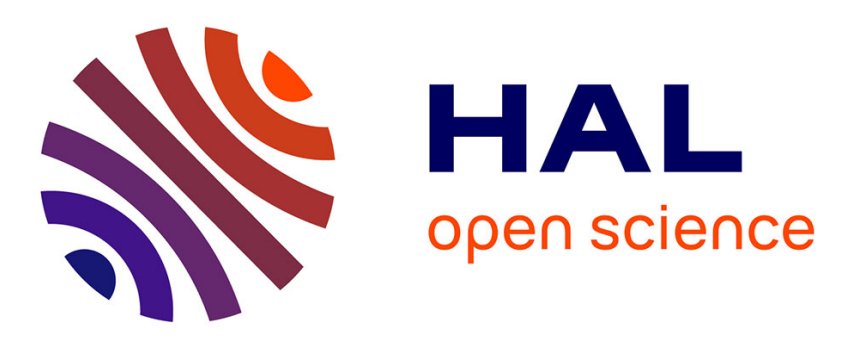

\title{
How tumor growth can be influenced by delayed interactions between cancer cells and the microenvironment?
}

Dibakar Ghosh, Subhas Khajanchi, Sylvain Mangiarotti, Fabrice Denis, Syamal K. Dana, Christophe Letellier

\section{To cite this version:}

Dibakar Ghosh, Subhas Khajanchi, Sylvain Mangiarotti, Fabrice Denis, Syamal K. Dana, et al.. How tumor growth can be influenced by delayed interactions between cancer cells and the microenvironment?. BioSystems, 2017, 158, pp.17 - 30. 10.1016/j.biosystems.2017.05.001 . hal-01672073

HAL Id: hal-01672073

https://hal-normandie-univ.archives-ouvertes.fr/hal-01672073

Submitted on 23 Dec 2017

HAL is a multi-disciplinary open access archive for the deposit and dissemination of scientific research documents, whether they are published or not. The documents may come from teaching and research institutions in France or abroad, or from public or private research centers.
L'archive ouverte pluridisciplinaire HAL, est destinée au dépôt et à la diffusion de documents scientifiques de niveau recherche, publiés ou non, émanant des établissements d'enseignement et de recherche français ou étrangers, des laboratoires publics ou privés. 


\section{Accepted Manuscript}

Title: How tumor growth can be influenced by delayed interactions between cancer cells and the microenvironment?

Author: Dibakar Ghosh Subhas Khajanchi Sylvain Mangiarotti Fabrice Denis Syamal K. Dana Christophe Letellier

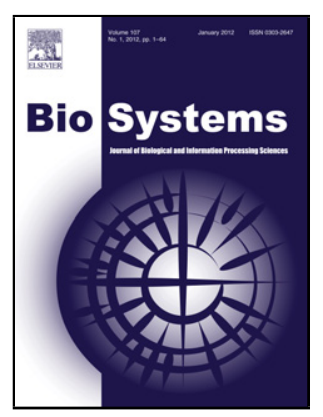

PII: S0303-2647(16)30276-3

DOI: http://dx.doi.org/doi:10.1016/j.biosystems.2017.05.001

Reference: BIO 3744

To appear in: BioSystems

Received date: 4-11-2016

Revised date: $17-4-2017$

Accepted date: 9-5-2017

Please cite this article as: Dibakar Ghosh, Subhas Khajanchi, Sylvain Mangiarotti, Fabrice Denis, Syamal K. Dana, Christophe Letellier, How tumor growth can be influenced by delayed interactions between cancer cells and the microenvironment?, <![CDATA[BioSystems]]> (2017), http://dx.doi.org/10.1016/j.biosystems.2017.05.001

This is a PDF file of an unedited manuscript that has been accepted for publication. As a service to our customers we are providing this early version of the manuscript. The manuscript will undergo copyediting, typesetting, and review of the resulting proof before it is published in its final form. Please note that during the production process errors may be discovered which could affect the content, and all legal disclaimers that apply to the journal pertain. 


\title{
How tumor growth can be influenced by delayed interactions between cancer cells and the microenvironment?
}

\author{
Dibakar Ghosh \\ Physics and Applied Mathematics Unit, Indian Statistical Institute, 203, B. T. Road, Kolkata, 700108, India \\ Subhas Khajanchi \\ Department of Mathematics, Bankura University, West Bengal - 722155, India \\ Sylvain Mangiarotti \\ Centre d'Études Spatiales de la Biosphère, CNRS-UPS-CNES-IRD, Observatoire Midi-Pyrénées, 18 avenue E. Belin, \\ 31400 Toulouse, France
}

Fabrice Denis

Centre Jean Bernard, 9 rue Beauverger, 72000 Le Mans, France

Syamal K. Dana

CSIR-Indian Institute of Chemical Biology, Kolkata 700032, India

Christophe Letellier

Normandie University - CORIA, Avenue de l'Université, F-76800 Saint-Etienne du Rouvray, France

\begin{abstract}
If recent advances in oncology emphasized the role of microenvironment in tumor growth, the role of delays for modeling tumor growth is still uncertain. In this paper, we considered a model, describing the interactions of tumor cells with their microenvironment made of immune cells and host cells, in which we inserted, as suggested by the clinicians, two time delays, one in the interactions between tumor cells and immune cells and, one in the action of immune cells on tumor cells. We showed analytically that the singular point associated with the co-existence of the three cell populations loses its stability via a Hopf bifurcation. We analytically calculated a range of the delays over which tumor cells are inhibited by immune cells and over which a period- 1 limit cycle induced by this Hopf bifurcation is observed. By using a global modeling technique, we investigated how the dynamics observed with two delays can be reproduced by a similar model without delays. The effects of these two delays was thus interpreted in terms of interactions between the cell populations.
\end{abstract}

Keywords: Cancer model, Tumor micro-environment, Delayed system, Chaos, Template.

\footnotetext{
${ }^{1}$ E-mail:dibakar@isical.ac.in
} 


\section{Introduction}

Cancer or malignant tumor is a world-wide problem, mainly because the underlying mechanism of tumor growth is not well understood and, consequently, is quite unpredictable and challenging to control it $[1,2,3,4$. The malignant tumor invades surrounding tissues and primarily grows in the mesenchyme;

5 it has the capability to grow in distant organs once the angiogenic switch occurred, leading to the formation of metastases. Interactions between tumors and their environments not only induce genetic instability of cancer cells but also governs their proliferation [5]. The tumor growth is not always very fast: an initial tumor may remain confined to a very limited size below a detectable threshold for a long time by routine imaging; this is designated as "tumor dormancy" [6]. Indeed, the sole presence of mutant cells does not necessarily induce a quick proliferation of tumor cells leading to a deleterious cancer. Interactions of tumor cells with immune cells and host cells play an important role in cancer proliferation [7] which remains to be clarified. Most of the past mathematical studies were devoted to the role of the immune system [8, 9] and the the action of some chemotherapy, surgery, radiotherapy or hormonotherapy on tumor growth [10, 11, 12, 13, 14, 15, 16]. The role of the proximal environment the healthy (host) cells - of the tumor was more rarely considered [14, 17, 18, 19. In these last studies, the key point was that the role of host cells was taken into account as clinically suggested [20, 21, 22, Such an approach still needs further attention.

When delays in the interactions between tumor cells and their environment were considered in models, most often they corresponded to delays between the phases of the cell cycle affecting cells productions, proliferation and differentiation [13, 23, 24, 25, 26, 27, 28, 29, 30. For instance, it was shown that a delay, introduced in the tumor cells response to changes in their environment, affects proliferation of the former: shorter the delay, stronger the tumor [24]. Although it is not certain if the delay actually plays any significant role from the clinical point of view, it helps to understand that tumor cells are difficult to eradicate due to the speed with which they can respond to any change in their environment, including therapies [5, 21, 22, 31, 32. This paper deals with the interactions between tumor cells and their surrounding microenvironment (including the immune system), mainly emphasizing the role of host cells and considering the effect of delays in these interaction processes. We started from the model developed by de Pillis and Radunskaya [14] which can produce chaotic behaviors [17, 18]. The sensitivity to initial conditions of such behaviors easily matches with clinical observations. Our objective is not to investigate a model describing in a quite exhaustive way all phenomena at the cell level but rather a qualitative model working at the tissue level. However, in the original model [14, 17, 18, the immune system was assumed to respond instantaneously to the presence of tumor cells. Since there is an obvious delay in the response to the presence of tumor cells, as suggested by clinical evidence that antitumor or activity by immunotherapy is not observed instantaneously but 2 to 10 weeks later after the initiation of 
a treatment [33, we modified the original model [14] by adding two time delays in the action of tumor cells on immune cells and, of immune cells on tumor cells. The presence of delays in nonlinear dynamical systems always affects the stability of the singular (equilibrium) points and, in particular, affecting the Hopf bifurcation [34, 35] observed before more complex dynamics such as chaos [23, 36, 37, 38].

The subsequent part of this paper is organized as follows. Section 2 is devoted to a brief presentation of the delay differential equations governing the interactions between host, immune and tumor cells that we investigated. In Section 3, an analytical study of the model is performed (stability of the singular points, persistence of limit cycle, etc.) and a numerical validation of our analytical results is discussed. In Section 4 we numerically investigated how this cancer dynamics is affected by our two time delays. In section 5, we employ the technique of global modeling to study the equivalence of the model without delay. Section 6 provides a discussion of our results.

\section{The Model}

Over the last few decades many models have been proposed for understanding the dynamics of cancer-immune interactions but a very few of them includes the host (healthy) cells. In their model, de Pillis and Radunskaya 14] considered that the immune and the tumor cells were also interacting with the host cells (Fig. 1). However, they assumed that all the interactions were instantaneous. As suggested by some clinical evidences of delayed interactions [39, 40], we introduced two time delays, one in the action of tumor cells on effector cells and one, in the action of effector cells on tumor cells. The model as proposed in [14] is thus modified in the set of three delay differential equations

$$
\left\{\begin{aligned}
\dot{E} & =\frac{\rho T E}{g+T}-\beta_{1} T\left(t-\tau_{1}\right) E\left(t-\tau_{1}\right)-\delta E \\
\dot{H} & =\alpha H\left(1-\frac{H}{k_{1}}\right)-\gamma_{1} T H \\
\dot{T} & =a T\left(1-\frac{T}{k_{2}}\right)-\beta_{2} T\left(t-\tau_{2}\right) E\left(t-\tau_{2}\right)-\gamma_{2} T H
\end{aligned}\right.
$$

where $E(t), H(t)$ and $T(t)$ designate the population of activated effector cells, host cells and tumor cells at any time $t$, respectively. In the first equation of system (1), the first term describes the proliferation enhancement of tumor-specific effector cells by tumor cells using a Michaelis-Menten type saturation of the immune system where $\rho$ is the rate of proliferation and $g$ is the value at which the growth rate of effector immune cells is half its maximum value. The term $-\beta_{1} T\left(t-\tau_{1}\right) E\left(t-\tau_{1}\right)$ corresponds to the inhibition of immune effector cells by tumor cells at rate $\beta_{1}$. The third term represents the effector cell natural death with a corresponding mean half-life $1 / \delta$. The second equation in system (1) represents the dynamics of host cells where the first term designates the Logistic growth of host cells in which $\alpha$ is the intrinsic growth rate and $k_{1}$ the biotic capacity. The competition between tumor and host cells obeys the law of mass action, here described as $\gamma_{1} T H$ where $\gamma_{1}$ is the inhibition rate. The third equation of 


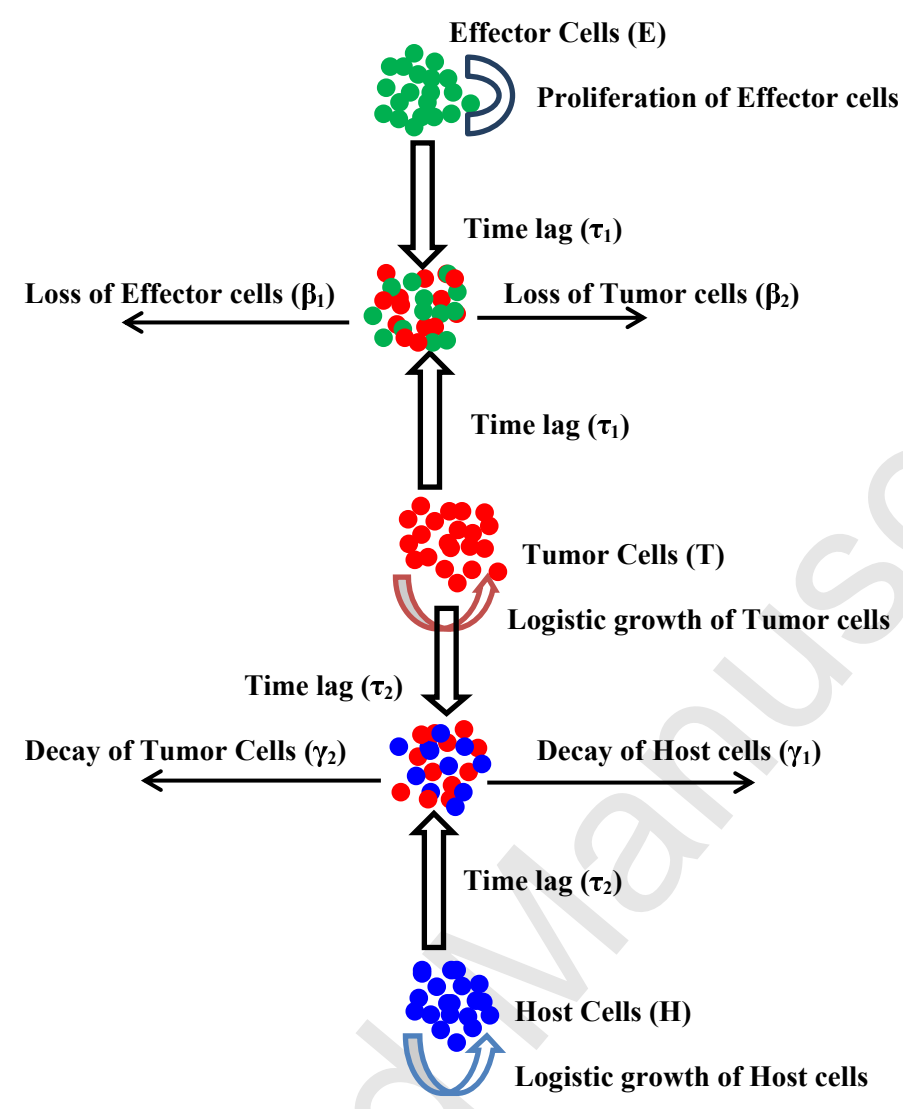

Figure 1: (Color online) Schematic diagram of model 11 where effector, tumor and host cells are in green (gray), red(light black) and blue(black), respectively.

system (1) represents the rate of change in tumor cells where the first term is the logistic growth of tumor 65 cells $a T\left(1-\frac{T}{k_{2}}\right)$, in absence of immune action depending on a growth rate $a$ and the environmental carrying capacity $k_{2}$. Interactions between tumor and effector cells are described by the degradation term $-\beta_{2} T\left(t-\tau_{2}\right) E\left(t-\tau_{2}\right)$ of the formers by the latter at rate $\beta_{2}$. The last term $\gamma_{2} T H$ represents the competition between tumor cells and host cells. The role of vascularization could have been taken into account as in 41, 19 but this would have increased the dimensionality of the model under consideration (since endothelial cells would have to be included as in [19]). Our mathematical investigations would have been overcomplicated in an undue way since our objective is to investigate the role of delays in tumor growth and, in particular, in which situations it would be required to introduce them in such a model.

Effector cells are the most relevant cells in the immune system which is distributed throughout our bodies and provide the main defense mechanism against pathogenic microorganism, virally infected cells and tumor cells. Effector cells mainly kill tumor cells by a two stage process: i) they deliver biochemical 
signals to tumor cells and, ii) they bind the membrane of cancerous cells. When effector cells form tumoreffector complexes, effector cells emanate soluble diffusible chemicals (known as "chemokines") which mobilize more and more effector cells around the neighborhood of malignant tumor cells and destroy them. An optimal antitumor efficiency is obtained when there is an activation of different effector cells (co-stimulation of $\mathrm{T}$ cells and accessory cells) [42. However, the formation of such a three-cell complex and the destruction of tumor cells is not an instantaneous process and few hours are required to detect the expression of some cytokine whose presence is required for activating the immune cells. This thus justifies our introduction of a time delay $\tau_{2}$ in the term describing the killing of tumor cells by the effector cells in the third equation of system (1). The tumor microenvironment is rich in cytokines and other inflammatory mediators which can be exploited by tumor cells for their growth and development [43. Since tumor cells may develop multiple resistance mechanisms including local immune suppression, induction of tolerance and systemic dysfunction in T-cell signaling [44, 45, 46, they have the ability to inhibit immune cells making the immune system ineffective. Moreover, tumors may exploit several distinct pathways such as the Program Death-1 (PD1) checkpoint to actively avoid their own destruction by the immune cells. Such a immuno-suppressed environment is not instantaneously obtained and one might consider a time delay $\tau_{1}$ before which tumor cells reduce the activity of immune cells, that is, reduce the population of effector cells. This time delay is introduced in the term describing the killing of effector cells by tumor cells in the first equation of system (1). Tumor cells are known to have a faster dynamics than other types of cells [5, 31, 21, 32, 22 and, consequently, the delay with which tumor cells act on effector cells is assumed shorter than the delay with which effector cells respond to the presence of tumor cells. Commonly, we should have thus $0 \leq \tau_{1}<\tau_{2}$.

For the sake of convenience and for removing numerical stiffness in the dynamics of system (1) we renormalized the state variables according to

$$
(x, y, z)=\left(\frac{E}{g}, \frac{H}{k_{1}}, \frac{T}{k_{2}}\right)
$$

and $\bar{t}=a t$ where $x$ designates the normalized population of effector cells, $y$ the population of host cells and $z$ the tumor population. We redefine the set of parameters by

$$
\left(\bar{\rho}, \bar{g}, \bar{\beta}_{1}, \bar{\delta}, \bar{\alpha}, \bar{\gamma}_{1}, \bar{\beta}_{2}, \bar{\gamma}_{2}\right)=\left(\frac{\rho}{a}, \frac{g}{k_{2}}, \frac{\beta_{1} k_{2}}{a}, \frac{\delta}{a}, \frac{\alpha}{a}, \frac{\gamma_{1} k_{2}}{a}, \frac{\beta_{2} g}{a}, \frac{\gamma_{2} k_{1}}{a}\right) .
$$

The normalized dynamical system (1) is

$$
\left\{\begin{array}{l}
\dot{x}=\frac{\rho x z}{g+z}-\beta_{1} x\left(t-\tau_{1}\right) z\left(t-\tau_{1}\right)-\delta x \\
\dot{y}=\alpha y(1-y)-\gamma_{1} y z \\
\dot{z}=z(1-z)-\beta_{2} x\left(t-\tau_{2}\right) z\left(t-\tau_{2}\right)-\gamma_{2} y z
\end{array}\right.
$$


System (4) is closed by choosing initial conditions $\psi=\left(\psi_{1}, \psi_{2}, \psi_{3}\right)$ defined in the space

$$
\begin{aligned}
S_{+} & =\left\{\psi \in S\left([-\xi, 0] \in \mathbb{R}_{+}^{3}\right): x(\xi)=\psi_{1}(\xi),\right. \\
y(\xi) & \left.=\psi_{2}(\xi), z(\xi)=\psi_{3}(\xi)\right\}
\end{aligned}
$$

where $\xi=\left[-\max \left\{\tau_{1}, \tau_{2}\right\}, 0\right], \psi_{i}(\xi) \geq 0(i=1,2,3)$ and $\psi_{i}$ are continuous functions on the interval $100\left[-\max \left\{\tau_{1}, \tau_{2}\right\}, 0\right]$ that may display jumps at $\xi=0$.

\section{General Properties and stability Analysis}

Our investigation of the delay differential system (4) starts with its positive invariance. System (4) is rewritten as

$$
\dot{\mathbf{X}}=\mathcal{M}(\mathbf{X})
$$

with $\mathbf{X}=(x, y, z)^{T} \in \mathbb{R}_{+}^{3}$ and

$$
\mid \begin{aligned}
\mathcal{M}_{1}(\mathbf{X})= & \frac{\rho x z}{g+z}-\beta_{1} x\left(t-\tau_{1}\right) z\left(t-\tau_{1}\right)-\delta x \\
\mathcal{M}_{2}(\mathbf{X})= & \alpha y(1-y)-\gamma_{1} y z \\
\mathcal{M}_{3}(\mathbf{X})= & z(1-z)-\beta_{2} x\left(t-\tau_{2}\right) z\left(t-\tau_{2}\right) \\
& -\gamma_{2} y z
\end{aligned}
$$

where $\mathcal{M} \in C^{\infty}\left(\mathbb{R}_{+}^{3}\right)$ is defined in the positive quadrant $\mathbb{R}_{+}^{3}$ and the mapping $\mathcal{M}: S_{+} \mapsto \mathbb{R}^{3}$. The right hand side of system (7) is locally Lipschitz - meaning that the derivatives are bounded - and satisfies

$$
\left.\mathcal{M}_{i}(\mathbf{X})\right|_{Y_{i}(t)}, \mathbf{X} \in S_{+}=\mathcal{M}_{i}(\mathbf{0}) \quad(i=1,2,3) .
$$

According to the second lemma in 47, every solution of system (4) with the initial conditions (5), $\Psi_{i}(t) \in S_{+}$, say $\mathbf{Y}(t)=\mathbf{Y}(t ; \mathbf{Y}(0))$, for all $t>0$, that is, it remains positive throughout the domain $S_{+}$, $\forall t>0$.

Proposition 3.1. For any positive initial function $\psi_{i}(i=1,2,3)$ which is continuous on $\left[-\max \left\{\tau_{1}, \tau_{2}\right\}, 0\right]$, there exist nonnegative solutions to system (4) which are bounded for all positive time.

Proof: The local existence and uniqueness of any solution to system (4) on the finite interval $\left[-\max \left\{\tau_{1}, \tau_{2}\right\}, 0\right]$ is a consequence of some properties as follows. Since the right-hand side of system (4) is $C^{1}$ (class of continuously differentiable functions) satisfying the properties of locally Lipschitz 110 functions, the existence and uniqueness of solution to system (4) is guaranteed according to the CauchyLipschitz theorem 48, 49]. 
Let us now consider the first equation of system (4). From the nonnegativity of its solutions, the right-hand side of system (4) is bounded by

$$
\frac{d x}{d t} \leq \frac{\rho x z}{g+z}-\delta x
$$

Hence, $x(t) \leq \max \left\{0, \varphi_{1}(0)\right\}$ if $\frac{\rho}{\delta}<1$. From the second equation and since solutions are bounded in the positive octant, the right-hand side is bounded by the Logistic growth term $\alpha y(1-y)$. Hence, $y(t) \leq \max \left\{\varphi_{2}(0), 1\right\}$. In a similar way, the third equation leads to $z(t) \leq \max \left\{\varphi_{3}(0), 1\right\}$. Therefore, solutions to system (4) are positive and bounded for any time $t \in \mathbb{R}^{+}$.

\subsection{Singular points}

System (4) has six singular points in the positive octant (the single ones which have a biological interest).

1. The singular point $E_{0}(0,0,0)$ is located at the origin of the state space.

2. The tumor-free singular point $E_{1}(0, \bar{y}, 0)$ where $\bar{y}=1$.

3. The tumor singular point $E_{2}(0,0, z)$ where $z=1$ which is characterized by the fact that effector and host cells are not present.

4. The host-free singular point $E_{3}$ whose coordinates are

$$
\mid \begin{aligned}
& \hat{x}=\frac{1-\hat{z}}{\beta_{2}} \\
& \hat{y}=0 \\
& \hat{z}=\frac{\left(\rho-\delta-g \beta_{1}\right)+\sqrt{\left(\rho-\delta-g \beta_{1}\right)^{2}-4 g \delta \beta_{1}}}{2 \beta_{1}}
\end{aligned}
$$

where the third coordinate $\hat{z}$ is a solution to the quadratic equation

$$
\beta_{1} \hat{z}^{2}+\hat{z}\left(\delta+g \beta_{1}-\rho\right)+g \delta=0 .
$$

Two real positive roots exist if $\frac{\delta}{g}+\beta_{1}<\frac{\rho}{g}$ and $\hat{z}<1$. When $\hat{z}=1$, the singular point $E_{3}$ merges with the tumor singular point $E_{2}$ (the tumor always persists in that case).

5. The effector-free singular point

$$
E_{4}=\mid \begin{aligned}
& \breve{x}=0 \\
& \breve{y}=\frac{\alpha-\gamma_{1}}{\alpha-\gamma_{1} \gamma_{2}} \\
& \breve{z}=\frac{\alpha\left(1-\gamma_{2}\right)}{\alpha-\gamma_{1} \gamma_{2}}
\end{aligned}
$$

exists when $\alpha \neq \gamma_{1} \gamma_{2}$. The effector-free singular point is in the positive octant if $\alpha>\gamma_{1}, \gamma_{2}<1$, and $\alpha>\gamma_{1} \gamma_{2}$. If $\gamma_{2}=1$, this singular point $E_{4}$ merges with the tumor-free singular point $E_{1}$. 
6. The three-cell singular point $E^{*}$ whose coordinates are

$$
\mid \begin{aligned}
x^{*} & =\frac{\alpha\left(1-\gamma_{2}\right)+z^{*}\left(\gamma_{1} \gamma_{2}-\alpha\right)}{\alpha \beta_{2}} \\
y^{*} & =\frac{\alpha-\gamma_{1} z^{*}}{\alpha} \\
z^{*} & =\frac{\left(\rho-\delta-g \beta_{1}\right) \pm \sqrt{\left(\rho-\delta-g \beta_{1}\right)^{2}-4 g \delta \beta_{1}}}{2 \beta_{1}}
\end{aligned}
$$

exists in the positive octant if

$$
\left\{\begin{array}{l}
\rho>\min \left\{\delta+g \beta_{1}, \delta+g \beta_{1}+2 \sqrt{g \delta \beta_{1}}\right\} \\
\gamma_{2}<1 \\
\gamma_{1} \gamma_{2}>\alpha \\
z^{*}<\frac{\alpha}{\gamma_{1}}
\end{array}\right.
$$

These conditions are hereafter designated as conditions (H1).

$$
\text { effector-free s }
$$

\subsection{Local Stability and Hopf bifurcation}

We focus here on the local stability of the biological meaningful singular points, that is, the singular points in the positive octant. Since delays $\tau_{1}$ and $\tau_{2}$ do not affect neither the number nor the type of singular points, we first investigate the local stability of the non-delayed system, assuming, $\tau_{1}=\tau_{2}=0$. We compute the Jacobian matrix of system (4) at each of the singular points,

$$
J_{E}=\left[\begin{array}{ccc}
\frac{\rho z}{g+z}-\delta-\beta_{1} z & 0 & \frac{g \rho x}{(g+z)^{2}}-\beta_{1} x \\
0 & \alpha(1-2 y)-\gamma_{1} z & -\gamma_{1} y \\
-\beta_{2} z & -\gamma_{2} z & 1-2 z-\gamma_{2} y-\beta_{2} x
\end{array}\right]
$$

At singular point $E_{0}$, the eigenvalues of the Jacobian $J_{E_{0}}$ at the origin are

$$
\Lambda^{0}=\mid \begin{aligned}
& \lambda_{1}^{0}=-\delta<0 \\
& \lambda_{2}^{0}=\alpha>0 \\
& \lambda_{3}^{0}=1>0 .
\end{aligned}
$$

$E_{0}$ thus represents a saddle point with a two-dimensional unstable manifold in the $y$ - $z$ plane and a 135 one-dimensional stable manifold along the $x$-axis. So, there is no initial condition outside the positive octant that can converge to $E_{0}$. 
The eigenvalues associated with the tumor-free singular point $E_{1}$ are

$$
\Lambda^{1}=\mid \begin{gathered}
\lambda_{1}^{1}=-\delta<0, \\
\lambda_{2}^{1}=-\alpha<0, \\
\lambda_{3}^{1}=1-\gamma_{2} .
\end{gathered}
$$

The singular point $E_{1}$ is a stable node when $\lambda_{3}^{1}<0$ and if $\gamma_{2}>1$; otherwise this singular point is a saddle point. Note that when the tumor-free singular point $E_{1}$ is a stable node, the effector-free singular point $E_{4}$ and the three-cell singular points $E^{*}$ do no longer exist. In other words, when the parameter values are such as the tumor-free state is a point attractor, there no longer exists a possibility of a sustained tumor growth, the microenvironment is not conducive to tumor growth.

The eigenvalues associated with the tumor singular point $E_{2}$ are

$$
\Lambda^{2}=\mid \begin{aligned}
& \lambda_{1}^{2}=\frac{\rho}{g+1}-\beta_{1}-\delta \\
& \lambda_{2}^{2}=\alpha-\gamma_{1} \\
& \lambda_{3}^{2}=-1<0
\end{aligned}
$$

When $\rho<\left(\beta_{1}+\delta\right)(g+1)$ and $\alpha<\gamma_{1}$, that is, when immune and host cells are not sufficiently proliferating to compete for colonizing the site, tumor cells remain the sole cells in the site: the tumor singular point is a stable node. Otherwise, when the growth rates of the effector and the host cells are strong enough to resist the tumor cell proliferation, this singular point is a saddle point, which is thus an impossible state to reach. The tumor cannot grow very quickly since tumor cells remain in competition with the micro-environment.

The eigenvalues associated with the host-free singular point $E_{3}$ are

$$
\Lambda^{3}=\mid \begin{aligned}
& \lambda_{1}^{3}=\alpha-\gamma_{1} \hat{z} \\
& \lambda_{2,3}^{3}
\end{aligned}
$$

where $\lambda_{2,3}^{3}$ are the roots of the characteristics equation

$$
\lambda^{2}+p_{1} \lambda+p_{2}=0
$$

with

$$
\left\{\begin{array}{l}
p_{1}=\delta+\beta_{1} \hat{z}+\beta_{2} \hat{x}+2 \hat{z}-1-\frac{\rho \hat{z}}{g+\hat{z}}, \\
p_{2}=\left(\frac{\rho \hat{z}}{g+\hat{z}}-\beta_{1} \hat{z}-\delta\right)\left(1-\beta_{2} \hat{x}-2 \hat{z}\right)+\beta_{2} \hat{z}\left(\frac{g \rho \hat{x}}{(g+\hat{z})^{2}}-\beta_{1} \hat{x}\right)
\end{array}\right.
$$

that can be reduced to

$$
\left\{\begin{array}{l}
p_{1}=\delta+\beta_{1}+1-\frac{\rho}{g+1} \\
p_{2}=-\left(\frac{\rho}{g+1}-\beta_{1}-\delta\right)+\beta_{2}
\end{array}\right.
$$


since $\hat{x}=0$ and $\hat{z}=1$. The host-free singular point is a stable node point when $p_{1}>0, p_{2}>0$ and $\gamma_{1}>\alpha$, that is, when

$$
\left\{\begin{array}{l}
\rho<\left(\delta+\beta_{1}+1\right)(g+1) \\
\rho<\left(\delta+\beta_{1}\right)(g+1),
\end{array}\right.
$$

respectively. Such a configuration arises when the immune system is not sufficiently powerful. Contrary to this, when $p_{1}<0, p_{2}<0$ and $\gamma_{1}<\alpha$, the host-free singular point is a saddle point.

One of the eigenvalues associated with the effector-free singular point $E_{4}$ is

$$
\lambda_{1}^{4}=\frac{\rho \breve{z}}{g+\breve{z}}-\beta_{1} \breve{z}-\delta ;
$$

the other two eigenvalues $\lambda_{2,3}^{4}$ are the roots of the characteristic equation

$$
\lambda^{2}+q_{1} \lambda+q_{2}=0
$$

where

$$
\left\{\begin{array}{l}
q_{1}=2 \breve{z}+\gamma_{2} \breve{y}-\alpha \breve{y}-1 \\
q_{2}=\alpha \breve{y}\left(2 \breve{z}+\gamma_{2} \breve{y}-1\right)-\gamma_{1} \gamma_{2} \breve{y} \breve{z} .
\end{array}\right.
$$

According to the Routh-Hurwitz criterion, the singular point $E_{4}$ is stable if $q_{1}>0, q_{2}>0$ and $\lambda_{1}^{4}<0$. The latter equality implies that

$$
\rho \alpha\left(\gamma_{2}-1\right)\left(\gamma_{1} \gamma_{2}-\alpha\right)<\alpha\left(\beta_{1} g+\delta\right)\left(\gamma_{2}-1\right)\left(\gamma_{1} \gamma_{2}-\alpha\right)+\alpha^{2} \beta_{1}\left(\gamma_{2}-1\right)^{2}+\delta g\left(\gamma_{1} \gamma_{2}-\alpha\right)^{2},
$$

and $\lambda_{2,3}^{4}<0$ is obtained when

$$
\gamma_{2}\left(\alpha+\gamma_{1}\right)>\left(\alpha+\gamma_{1} \gamma_{2}^{2}\right)
$$

and

$$
\frac{\alpha+\gamma_{2}}{1+\gamma_{1}}>1
$$

The most interesting situation for an accurate understanding of tumor growth is to investigate its interaction with the microenvironment [7, 50, 51], that is, in our case, to investigate the singular point $E^{*}$ where effector, host and tumor cells are co-existing. For that reason, we took into account the impact of the two delays $\tau_{1}$ and $\tau_{2}$ on the stability of this singular point. In order to do that, we linearized system (4) at $E^{*}$ in the form

$$
\frac{d X}{d t}=M X(t)+N X\left(t-\tau_{1}\right)+P X\left(t-\tau_{2}\right)
$$

where

$$
M=\left[\begin{array}{ccc}
\frac{\rho z^{*}}{g+z^{*}}-\delta & 0 & \frac{g \rho x^{*}}{\left(g+z^{*}\right)^{2}} \\
0 & -\alpha y^{*} & -\gamma_{1} y^{*} \\
0 & -\gamma_{2} z^{*} & 1-2 z^{*}-\gamma_{2} y^{*}
\end{array}\right],
$$




$$
N=\left[\begin{array}{ccc}
-\beta_{1} z^{*} & 0 & -\beta_{1} x^{*} \\
0 & 0 & 0 \\
0 & 0 & 0
\end{array}\right], P=\left[\begin{array}{ccc}
0 & 0 & 0 \\
0 & 0 & 0 \\
-\beta_{2} z^{*} & 0 & -\beta_{2} x^{*}
\end{array}\right]
$$

and $X(\cdot)=\left(x(\cdot), y(\cdot), z(\cdot)^{T}\right)$ is the state vector. The characteristic equation of the linearized system $(30)$ is

$$
\operatorname{det}\left(\lambda I-M-N e^{-\lambda \tau_{1}}-P e^{-\lambda \tau_{2}}\right)=0
$$

which can be explicitly expressed as

$$
D\left(\lambda, \tau_{1}, \tau_{2}\right) \equiv A(\lambda)+B(\lambda) e^{-\lambda \tau_{1}}+C(\lambda) e^{-\lambda \tau_{2}}=0
$$

where $K=\{A, B, C\}$ are three polynomials in $\lambda$ in the form

$$
K(\lambda)=\lambda^{3}+k_{1} \lambda^{2}+k_{2} \lambda+k_{3}
$$

Their coefficients are

$$
\begin{aligned}
& \left\{\begin{array}{l}
A_{1}=-1+2 z^{*}+\left(\gamma_{2}+\alpha\right) y^{*}+\left(\delta-\frac{\rho z^{*}}{g+z^{*}}\right) \\
A_{2}=\alpha y^{*}\left(-1+2 z^{*}+\gamma_{2} y^{*}\right)-\gamma_{1} \gamma_{2} y^{*} z^{*}+\left(-1+2 z^{*}+\left(\gamma_{2}+\alpha\right) y^{*}\right)\left(\delta-\frac{\rho z^{*}}{g+z^{*}}\right) \\
A_{3}=\alpha y^{*}\left[\left(-1+2 z^{*}+\gamma_{2} y^{*}\right)-\gamma_{1} \gamma_{2} y^{*} z^{*}\right]\left(\delta-\frac{\rho z^{*}}{g+z^{*}}\right),
\end{array}\right. \\
& \left\{\begin{array}{l}
B_{1}=\beta_{1} z^{*} \\
\left.B_{2}=\beta_{1} z^{*}\left(-1+2 z^{*}+\gamma_{2} y^{*}+\alpha\right) y^{*}\right) \\
B_{3}=\beta_{1} y^{*} z^{*}\left[\alpha\left(-1+2 z^{*}+\gamma_{2} y^{*}\right)-\gamma_{1} \gamma_{2} y^{*} z^{*}\right],
\end{array}\right.
\end{aligned}
$$

and

respectively.

$$
\left\{\begin{array}{l}
C_{1}=\beta_{2} x^{*} \\
C_{2}=\alpha \beta_{2} x^{*} y^{*}+\beta_{2} x^{*}\left(\delta-\frac{\rho z^{*}}{g+z^{*}}\right)+\frac{g \rho \beta_{2} x^{*} z^{*}}{\left(g+z^{*}\right)^{2}} \\
C_{3}=\alpha \beta_{2} x^{*} y^{*}\left(\delta-\frac{\rho z^{*}}{g+z^{*}}\right)+\alpha \beta_{2} y^{*} z^{*} \frac{g \rho x^{*}}{\left(g+z^{*}\right)^{2}}
\end{array}\right.
$$

The three-cell singular point $E^{*}$ is stable if the roots of the characteristic equation 32 have negative real parts. The classical Routh-Hurwitz criterion is not applicable to the delayed system (4) since equation (32) is a transcendental equation and has an infinite number of solutions. To determine the stability of $E^{*}$, we investigated the distribution of the roots of equation 32 with the help of a Lemma used by Ruan and Wei [52]. 
Lemma 3.2 Let be

$$
\begin{aligned}
P\left(\lambda, e^{-\lambda \tau_{1}}, e^{-\lambda \tau_{2}}, \ldots, e^{-\lambda \tau_{s}}\right)= & \lambda^{n}+p_{1}^{0} \lambda^{n-1}+p_{2}^{0} \lambda^{n-2}+\ldots+p_{n-1}^{0} \lambda+p_{n}^{0} \\
& +\left[p_{1}^{1} \lambda^{n-1}+\ldots+p_{n-1}^{1} \lambda+p_{n}^{1}\right] e^{-\lambda \tau_{1}}+\cdots \\
& +\left[p_{1}^{s} \lambda^{n-1}+\ldots+p_{n-1}^{s} \lambda+p_{n}^{s}\right] e^{-\lambda \tau_{s}}=0
\end{aligned}
$$

an exponential polynomial where $\tau_{i} \geq 0(i=0,1, \ldots, s)$ and $p_{i}^{j}(i=0,1, \ldots, s ; j=0,1, \ldots, n)$ are constants. As $\left(\tau_{1}, \tau_{2}, \ldots, \tau_{s}\right)$ vary, the sum of orders of the zeros of $P\left(\lambda, e^{-\lambda \tau_{1}}, e^{-\lambda \tau_{2}}, \ldots, e^{-\lambda \tau_{s}}\right)$ in the open right half plane can change only if a zero appears on or crosses the imaginary axis. According to this lemma, the stability analysis of the singular point can be performed by considering time delays as parameters of the considered system [52]. System (4) has two discrete time delays $\tau_{1}$ and $\tau_{2}$ and, consequently, it is necessary to limit the cases for investigating the roots of the transcendental equation 32. We thus limit ourselves to investigate the stability of the singular point $E^{*}$ for the following cases.

Case I: $\tau_{1}=\tau_{2}=0$. Without any delay, the characteristic equation (32) is reduced to

$$
\lambda^{3}+\underbrace{A_{1}+B_{1}+C_{1}}_{=e_{11}} \lambda^{2}+\underbrace{A_{2}+B_{2}+C_{2}}_{=e_{12}} \lambda+\underbrace{A_{3}+B_{3}+C_{3}}_{=e_{13}}=0
$$

If conditions (H1) hold then it can be easily shown that $e_{13}>0$. Consequently, according to the classical

Routh-Hurwitz criterion, all the roots of equation (37) have negative real part if

$$
e_{11}>0 \text { and } e_{11} e_{12}>e_{13}
$$

Therefore when $\tau_{1}=\tau_{2}=0$, the three-cell singular point $E^{*}$ is stable.

Case II: $\tau_{1}=0$ and $\tau_{2}>0$. The tumor cells instantaneously reduce the activity of immune cells but there is a delay before the immune system starts killing the tumor cells $\left(\tau_{2}>0\right)$. The characteristic equation 32 has the form

$$
\lambda^{3}+\left(A_{1}+B_{1}\right) \lambda^{2}+\left(A_{2}+B_{2}\right) \lambda+e^{-\lambda \tau_{2}}\left(C_{1} \lambda^{2}+C_{2} \lambda+C_{3}\right)=0
$$

This transcendental equation (38) has an infinite number of solutions according to Rouché's theorem [53. When $\tau_{2}$ is varied over $\mathbb{R}^{+}$, the sign of the roots of equation 38 changes when it crosses the imaginary axis. Since periodic solutions are also relevant to cancer dynamics (Jeff's phenomenon), it is useful to investigate the case where $\lambda=i \nu$. Once the imaginary parts are separated from real parts, we have

$$
\nu^{3}-\left(A_{2}+B_{2}\right) \nu=C_{2} \nu \cos \left(\nu \tau_{2}\right)-\left(C_{3}-C_{1} \nu^{2}\right) \sin \left(\nu \tau_{2}\right)
$$

and

$$
\left(A_{1}+B_{1}\right) \nu^{2}-\left(A_{3}+B_{3}\right)=C_{2} \nu \sin \left(\nu \tau_{2}\right)+\left(C_{3}-C_{1} \nu^{2}\right) \cos \left(\nu \tau_{2}\right)
$$


Squaring and adding 39 ) to 40 , we obtain

$$
\nu^{6}+m_{11} \nu^{4}+m_{12} \nu^{2}+m_{13}=0
$$

with

$$
\left\{\begin{array}{l}
m_{11}=\left(A_{1}+B_{1}\right)^{2}-2\left(A_{2}+B_{2}\right)-C_{1}^{2} \\
m_{12}=\left(A_{2}+B_{2}\right)^{2}-2\left(A_{1}+B_{1}\right)\left(A_{3}+B_{3}\right)+2 C_{1} C_{3}-C_{2}^{2} \\
m_{13}=\left(A_{3}+B_{3}\right)^{2}-C_{3}^{2} .
\end{array}\right.
$$

Let us assume that $\nu^{2}=s_{1}$, then (41) takes the form

$$
f\left(s_{1}\right)=s_{1}^{3}+m_{11} s_{1}^{2}+m_{12} s_{1}+m_{13}=0 .
$$

It is clear that

$$
m_{11}=\left(\delta+\beta_{1} z^{*}-\frac{\rho z^{*}}{g+z^{*}}\right)^{2}+\left(-1+2 z^{*}+\gamma_{2} y^{*}\right)^{2}+2 \gamma_{1} \gamma_{2} y^{*} z^{*}>0 .
$$

Now, $f(0)=m_{13}$ is negative if condition

$$
\alpha y^{*}\left(-1+2 z^{*}+\gamma_{2} y^{*}\right)+\gamma_{1} \gamma_{2} y^{*} z^{*}\left[\beta_{1} z^{*}-\left(\delta+\beta_{1} z^{*}-\frac{\rho z^{*}}{g+z^{*}}\right)\right]<\alpha \beta_{2} x^{*} y^{*}\left(\delta-\frac{\rho\left(z^{*}\right)^{2}}{\left(g+z^{*}\right)^{2}}\right)
$$

holds. Since $f\left(s_{1}\right) \mapsto \infty$ if $s_{1} \mapsto+\infty$, we can therefore assert that equation 43 has at least one nonnegative root. Without any loss of generality, we can conclude that equation (41) has a unique positive real root $\nu_{0}$ or, in other words, the characteristic equation (38) has purely imaginary roots $\pm i \nu_{0}$. Solving equations (39) and (40) to determine the critical value of $\tau_{2}$ for which system (4) remains stable, we get

$$
\tau_{2}^{j}=\frac{2 \pi j}{\nu_{0}}+\frac{1}{\nu_{0}} \arccos \left[\frac{C_{1}^{2}+\nu^{3}-\left(A_{2}+B_{2}\right) \nu}{\left(C_{1} \nu^{2}-C_{3}\right)^{2}+C_{2}^{2} \nu^{2}}-\frac{C_{3}\left[-\left(A_{1}+B_{1}\right) \nu^{2}+\left(A_{3}+B_{3}\right)\right]}{\left(C_{1} \nu^{2}-C_{3}\right)^{2}+C_{2}^{2} \nu^{2}}\right]
$$

with $j=0,1,2,3 \ldots$ Thus for $\tau_{2}=0$, the three-cell singular point $E^{*}$ is stable and due to Butler's lemma [54], it remains stable for every $\tau_{2}<\tau_{2}^{0}$. This means that with a delay beyond a given threshold, the competition between tumor cells and their micro-environment starts to lose its efficiency and, consequently, the proliferation of tumor cells is no longer strongly maintained, making possible fast tumor growth.

We now investigate the onset of a Hopf bifurcation [55] of system (4) when there is a pair of purely imaginary roots, and for which we need to verify the transversality condition

$$
\left.\frac{d \operatorname{Re}(\lambda)}{d \tau_{2}}\right|_{\tau_{2}=\tau_{2}^{(k)}}>0
$$

This indicates that there exists at least one eigenvalue whose real part is positive with $\tau_{2}>\tau_{2}^{(k)}$ and preserving the conditions for the existence of a periodic solution. First, we look for purely imaginary 
roots $\lambda=i \nu_{0}$ of the characteristic equation $(32)$ implying that $\left|A\left(i \nu_{0}\right)\right|=\left|B\left(i \nu_{0}\right)\right|$ determines the set of values for $\tau_{2}^{(k)}$. Our goal is to study how $\lambda$ evolves when $\tau_{2}$ is varied. In order to do that, we have to determine

$$
\prod=\left.\operatorname{sign}\left\{\frac{d \operatorname{Re}(\lambda)}{d \tau_{2}}\right\}\right|_{\tau_{2}=\tau_{2}^{(k)}}=\left.\operatorname{sign}\left\{\operatorname{Re} \frac{d \lambda}{d \tau_{2}}\right\}\right|_{\tau_{2}=\tau_{2}^{(k)}}
$$

by differentiating equation 38 with respect to $\tau_{2}$; we have thus

$$
\begin{gathered}
\frac{d \lambda}{d \tau_{2}}\left[3 \lambda^{2}+2\left(A_{1}+B_{1}\right) \lambda+\left(A_{2}+B_{2}\right)+\left(2 C_{1} \lambda+C_{2}-\tau_{2}\left(C_{1} \lambda^{2}+C_{2} \lambda+C_{3}\right)\right) e^{-\lambda \tau_{2}}\right] \\
=\lambda e^{-\lambda \tau_{2}}\left(C_{1} \lambda^{2}+C_{2} \lambda+C_{3}\right)
\end{gathered}
$$

implying that

$$
\left(\frac{d \lambda}{d \tau_{2}}\right)^{-1}=\frac{3 \lambda^{2}+2\left(A_{1}+B_{1}\right) \lambda+\left(A_{2}+B_{2}\right)}{\lambda e^{-\lambda \tau_{2}}\left(C_{1} \lambda^{2}+C_{2} \lambda+C_{3}\right)}+\frac{2 C_{1} \lambda+C_{2}}{\lambda\left(C_{1} \lambda^{2}+C_{2} \lambda+C_{3}\right)}-\frac{\tau_{2}}{\lambda} .
$$

A straightforward computation shows that the transversality condition is given by

$$
\left.\operatorname{Re}\left(\frac{d \lambda}{d \tau_{2}}\right)^{-1}\right|_{\lambda=i \nu_{0}}=\frac{1}{\nu_{0}^{2}}\left[\frac{2 \nu_{0}^{6}+\nu_{0}^{4}\left[\left(A_{1}+B_{1}\right)^{2}-2\left(A_{2}+B_{2}\right)-C_{1}^{2}\right]}{\left(C_{3}-C_{1} \nu_{0}^{2}\right)^{2}+C_{2}^{2} \nu_{0}^{2}}+\frac{C_{3}^{2}-\left(A_{3}+B_{3}\right)^{2}}{\left(C_{3}-C_{1} \nu_{0}^{2}\right)^{2}+C_{2}^{2} \nu_{0}^{2}}\right]>0 .
$$

Hence the transversality condition holds. We can sketch this result in the theorem as follows.

Theorem 3.2: If conditions (H1) and (H3) hold, then the three-cell singular point $E^{*}$ of system (4) presents a Hopf bifurcation for $\tau_{2}=\tau_{2}^{(k)}$. Furthermore, point $E^{*}$ is stable if $\tau_{2}<\tau_{2}^{(k)}$ and unstable if $\tau_{2}>\tau_{2}^{(k)}$.

Case III: $\tau_{1}>0, \tau_{2} \in\left[0, \tau_{2}^{(k)}\right)$. Now we shall study the influence of the two delays $\tau_{1}$ and $\tau_{2}$ on the stability of the singular point $E^{*}$. Without any loss of generality, we used the characteristic equation in the form of equation 32 where $\tau_{2}$ is locally asymptotically stable in $\left[0, \tau_{2}^{(k)}\right)$ and $\tau_{1}$ is considered as a parameter. First, we propose a result concerning the sign of real parts of the roots of equation 32 .

Proposition 3.2. For $\tau_{2}>0$, if all the roots of the characteristic equation (38) have negative real parts, then there exist $\tau_{1}\left(\tau_{2}\right)>0$, such that all the roots of $D\left(\lambda, \tau_{1}, \tau_{2}\right)=0$ have negative real part if $\tau_{1} \in\left[0, \tau_{1}\left(\tau_{2}\right)\right)$. [52]

Proof: Let us assume that equation (38) has no root with a positive real part for $\tau_{2} \in\left[0, \tau_{2}^{(k)}\right)$ and ${ }_{190} \tau_{1}=0$. In a similar way, equation (32) has no root with a positive real part for $\tau_{1}=0$ and $\tau_{2}>0$. By considering $\tau_{1}$ as a parameter and from the right member of equation (32), $D\left(\lambda, \tau_{1}, \tau_{2}\right)=0$ is analytic in $\lambda$ and $\tau_{1}$ when $\tau_{1}$ is varied. According to Theorem 2.1 by Ruan and Wei [52], when $\tau_{1}$ is varied, the sum of the multiplicity of the zeros of $D\left(\lambda, \tau_{1}, \tau_{2}\right)=0$ can vary in the right half-plane only if a zero occurs on or crosses the imaginary axis. Thus equation (32) with $\tau_{1}=0$ has no root with positive real part, then there exists $\tau_{1}\left(\tau_{2}\right)>0$ such as all roots of equation (32) with $\tau_{1} \in\left[0, \tau_{1}\left(\tau_{2}\right)\right.$ ) (meaning that $\tau_{1}$ depends on $\tau_{2}$ ) have negative real parts. 


\subsection{Domain of existence of the limit cycle}

We checked our analytical results with numerical simulations using a modified Heun method [56] for the parameter values reported in Table. 1 but setting $\rho=3.2$. Time delay $\tau_{1}$ is set to 0 and $\tau_{2}$ is used as the bifurcation parameter. The three-cell singular point $E^{*}$ located at $(0.1627,0.3901,0.2033)$ is such that $m_{11}=0.2776>0$ and $m_{13}=-0.0012<0$. There exists a single pure real root $\nu_{0}=0.2301$ when $\tau_{2}^{(0)}=0.0956$ and for which we found that $\left.\frac{d}{d \tau_{2}} \operatorname{Re} \lambda\left(\tau_{2}^{k}\right)\right|_{\tau_{2}=\tau_{2}^{(k)}}=0.5336>0$.

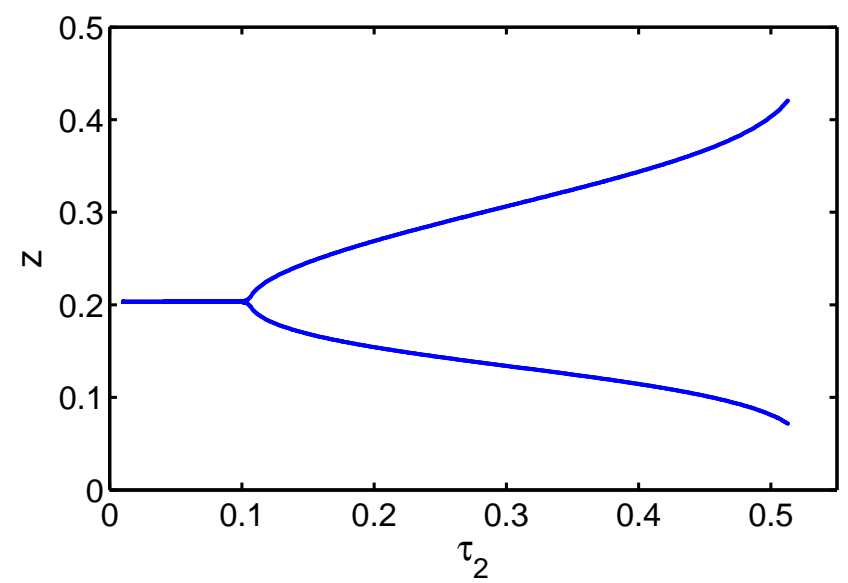

Figure 2: (Color online) Numerical bifurcation diagram of system (4) versus delay $\tau_{2}$ for $\rho=3.2, \tau_{1}=0$ and other parameter values as reported in Table 1 Numerically, the Hopf bifurcation occurs at $\tau_{2}=0.1031$ and the resulting period-1 limit cycle persists up to $\tau_{2}=0.5336$. For greater $\tau_{2}$ values, the trajectory is ejected to infinity.

Hence, according to Theorem 1 in Cooke and van den Driessche [57, it is clear that a bifurcation occurs when the value of $\tau_{2}$ is increased and in fact, it occurs at $\tau_{2}^{0}=0.0956$ as computed from equation 
Table 1: Symbols, biological meaning and numerical values of parameters from system 44. Parameter values of the global model 58 obtained without delay (see Section 5 are also reported.

\begin{tabular}{clcc}
\hline \hline Name & Description & With delays & Without \\
\hline \multicolumn{2}{c}{ maximum recruitment of effector cells by tumor cells } & 4.5 & 2.780 \\
$g$ & half saturation constant for the proliferation term & 1.0 & 1.0 \\
$\beta_{1}$ & inhibition rate of effector cells due to tumor cells & 0.2 & 0.0 \\
$\delta$ & decay rate of effector cells & 0.5 & 0.407 \\
$\alpha$ & intrinsic growth rate of host cells & 0.5 & 0.533 \\
$\gamma_{1}$ & host cells inactivation rate by tumor cells & 1.5 & 1.497 \\
$\beta_{2}$ & tumor cells inactivation rate by effector cells & 2.5 & 2.323 \\
$\gamma_{2}$ & inactivation rate of tumor cells due to host cells & 1.0 & 0.703 \\
\hline \hline
\end{tabular}

\section{Routes to chaos}

The system presents dynamical behaviors qualitatively matching with clinical observations [18. Our aim is now to investigate how the dynamics of system (4) evolves when one of the delay parameter values is varied. We selected the parameter values as reported in Tab. 11, with $\rho=4.5$ and $\tau_{1}=0.012 . \tau_{2}$ is selected as the bifurcation parameter. With the chosen parameter values, system (4) has five singular points in the positive octant, which are

$$
E_{0}=\left|\begin{array}{ll}
0 \\
0 \\
0
\end{array}, E_{1}=\right| \begin{aligned}
& 0 \\
& 1 \\
& 0
\end{aligned}, E_{2}=\left|\begin{array}{l}
0 \\
0 \\
1
\end{array}, E_{3}=\right| \begin{aligned}
& 0.34699 \\
& 0 \\
& 0.13250
\end{aligned} \quad \text { and } E^{*}=\mid \begin{aligned}
& 0.106002 \\
& 0.602491 \\
& 0.132503
\end{aligned} .
$$

By computing the roots of the characteristic equation (32), we found that $E_{0}$ is an unstable singular point, $E_{1}$ and $E_{2}$ are stable singular points under some parameter restrictions (they are unstable otherwise). Singular point $E_{3}$ is a saddle-focus with eigenvalues

$$
\Lambda_{3}=\mid \begin{aligned}
& +0.401 \\
& -0.06591 \pm 0.6129 i
\end{aligned}
$$

However, the three-cell singular point $E^{*}$ has eigenvalues

$$
\Lambda^{*}=\mid \begin{aligned}
& -0.5007 \\
& 0.0335 \pm 0.2622 i
\end{aligned}
$$




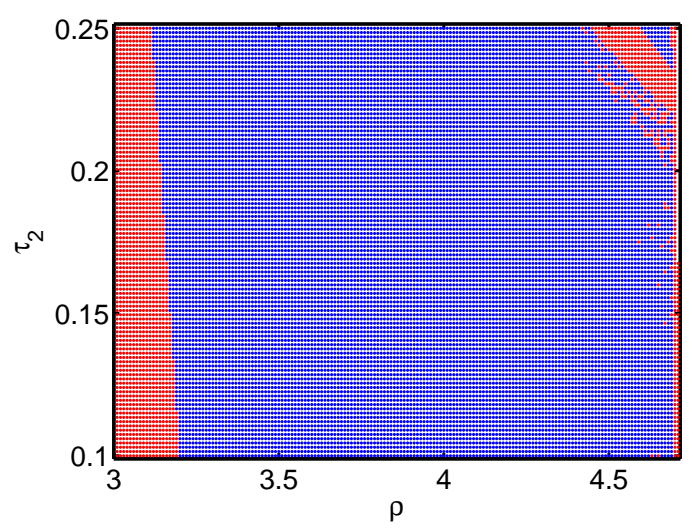

Figure 3: (Color online) Two-dimensional bifurcation diagram for the three-cell singular point $E^{*}$ of system (4). The recruitment of effector cells by tumor cells $\rho$ against the time delay $\tau_{2}$ is plotted. Other parameter values as reported in Tab. 1 and $\tau_{1}=0$. Colored regions show stability of the three-cell singular point $E^{*}$ : stable (red/gray) and unstable (blue/black).

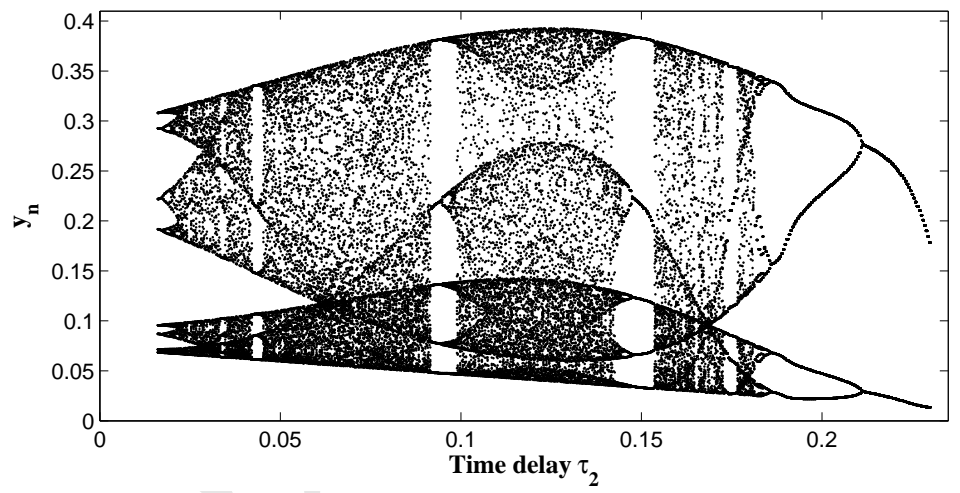

Figure 4: Bifurcation diagram of system (4) versus time delay $\tau_{2}$. Parameter values as reported in Tab. 1 . $\rho=4.5$, and $\tau_{1}=0.012$.

We numerically draw the bifurcation diagram in Fig. 4 for varying $\tau_{2}$ from 0.01 to 0.23 ; we used the Poincaré surface of section

$$
\mathcal{P} \equiv\left\{\left(x_{n}, y_{n}\right) \in \mathbb{R}^{2} \mid \dot{y}_{n}=0, \ddot{y}_{n}>0\right\}
$$

When $\tau_{2}$ is increased, there is a period-doubling cascade (here starting with a period-4 limit cycle) and leading to chaotic attractors. There is a period- 6 window $\left(\tau_{2}=0.044\right)$ and a period-5 window $\left(\tau_{2} \approx 0.092\right)$. Unstable periodic orbits are created up to $\tau_{2} \approx 0.123$. In the present case, the dynamics is the most developed when the two delays are nearly equal $\left(\tau_{1} \geq \tau_{2}\right)$. When the difference is increased, in the case $\tau_{1}>\tau_{2}$ as well as in the case $\tau_{2}>\tau_{1}$, the dynamics is reduced (unstable periodic orbits) up to low periodic limit cycle. Thus when $\tau_{2}$ is decreased, there is a period-doubling bifurcation $\left(\tau_{2} \approx 0.24\right.$, not shown) leading to a period-2 limit cycle (Fig. 5 a ) which is destabilized by a second period-doubling 


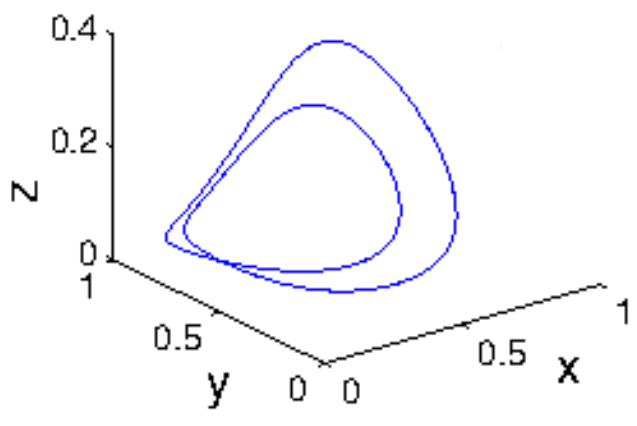

(a) $\tau_{2}=0.22$ : period-2 limit cycle

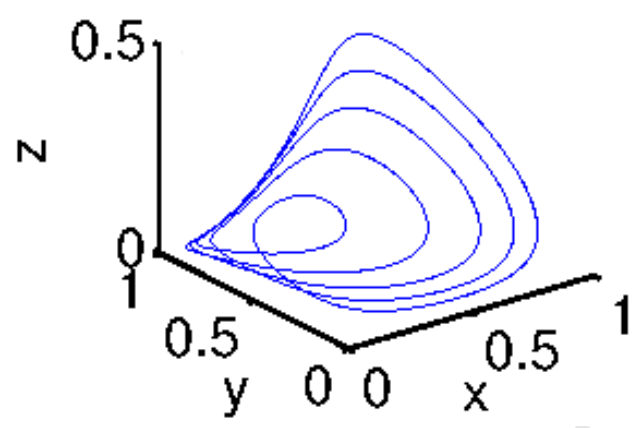

(c) $\tau_{2}=0.15$ : period-5 limit cycle

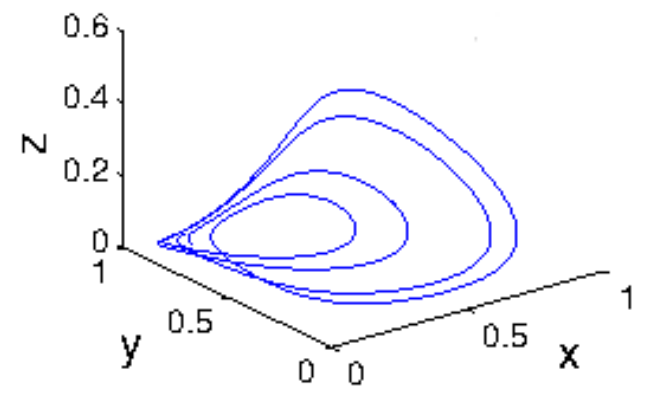

(b) $\tau_{2}=0.20$ : period-4 limit cycle

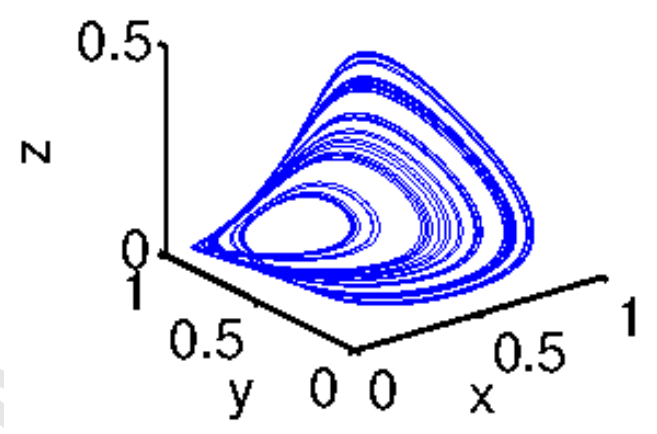

(d) $\tau_{2}=0.10$ : chaotic attractor

Figure 5: (Color online) Dynamical behaviors produced by system 4 for different values of the second time delay $\tau_{2}$. Initial conditions $x(0)=0.1, y(0)=0.2$, and $z(0)=0.5$. Parameter values as reported in Tab. 1 $\rho=4.5$ and $\tau_{1}=0.012$. of half-turns ( $\pi$-twists) and the decreasing branch, labeled by "1", is associated with a strip with an odd number of half-turns [60]. These two strips can be synthesized in the form of a branched manifold 


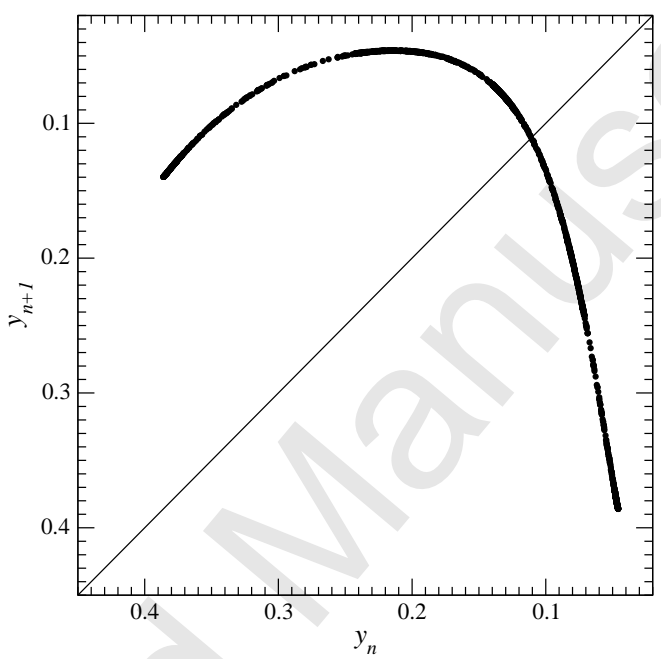

Figure 6: Smooth unimodal first-return map to a Poincaré section of the chaotic attractor observed for $\rho=4.5, \tau_{1}=0.012$ and $\tau_{2}=0.1$. Other parameter values as reported in Table 1

— also designated as template - made of a splitting chart where the two branches are separated, a socalled "mixer" where branches are twisted (local torsions) and permuted 61] and a branching line where branches are stretched and squeezed (see [61] for details about mixers and [60, 62] about templates, for instance).

Chaotic attractors are structured around a collection of unstable periodic orbits which can be used for characterizing their topology by computing topological invariants and for constructing templates [60, 62, 63. We thus compute linking numbers between couples of periodic orbits by counting oriented crossings in a regular plane projection, that is, in a plane projection where there is no more than two segments crossing at a given point 63. Period- $p$ orbits are designated by a so-called orbital sequence made of $p$ symbols $\sigma_{n}$ determined from the locations of their $p$ periodic points $y_{n}$ according to

$$
\sigma_{n}=\mid \begin{array}{cc}
0 & \text { if } y_{n}<y_{c} \\
1 & \text { otherwise. }
\end{array}
$$


where $y_{c}$ is the $y$-coordinate of the smooth maximum in the map shown in Fig. 6. As an example, the knot made of the period-2 orbit (10) and of the period-5 orbit (10110) is shown in Fig. 7. eight negative crossings were found thus leading to a linking number equal to -4 (the linking number is equal to the half-sum of the oriented crossings between segments of the two different orbits, self-crossings being ignored). Using many other linking numbers, we found that all of them are correctly predicted by a template described by the linking matrix

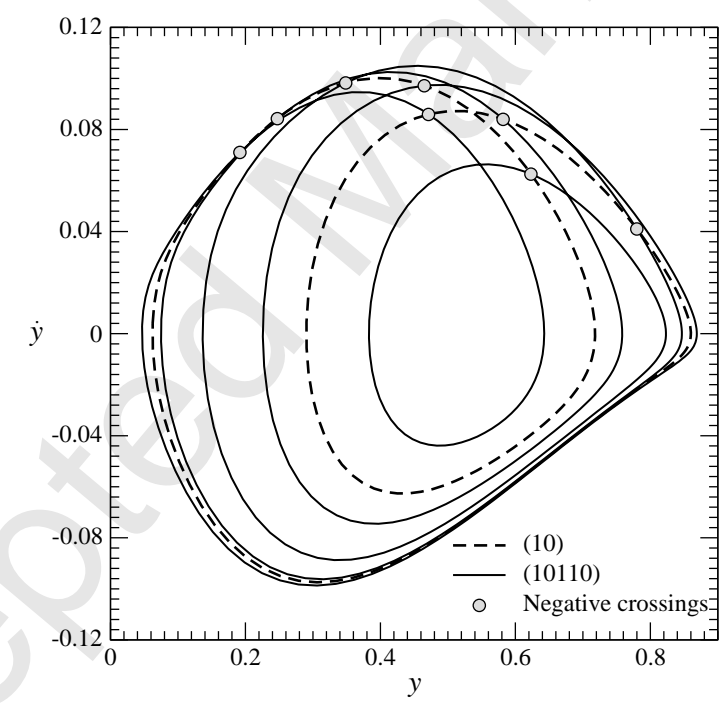

Figure 7: Knot made of the period-2 orbit (10) and of the period-5 orbit (10110) which were extracted from the chaotic attractor shown in Fig. 5 H. Eight negative crossings were counted on this regular plane projection.

$$
M_{i j}=\left[\begin{array}{cc}
0 & -1 \\
-1 & -1
\end{array}\right]
$$

where on-diagonal element $M_{i i}$ is associated with the local torsion applied to the $i$ th branch and offdiagonal element $M_{i j}(i \neq j)$ corresponds to the permutations between the $i$ th and the $j$ th branches [63, 60]. This linking matrix is sufficient to describe uniquely the template because we used the standard insertion convention at the branching line, according to which branches from back to front are ordered 
from left to right [63. The corresponding template is shown in Fig. 8 with the two periodic orbits (10) and (10110). Eight negative crossings - three induced by the local torsion applied to branch "1" and five induced by the permutation between the two branches - are found, thus leading to $1 \mathrm{k}(10,10110)=-4$ as counted in the regular plane projection shown in Fig. 7f the template correctly predicts the linking number $\operatorname{lk}(10,10110)$ as well as all the others we investigated, it is therefore validated. This accurate characterization of the dynamics will allow us to show that we are able to reproduce correctly the dynamics with a system without delays.

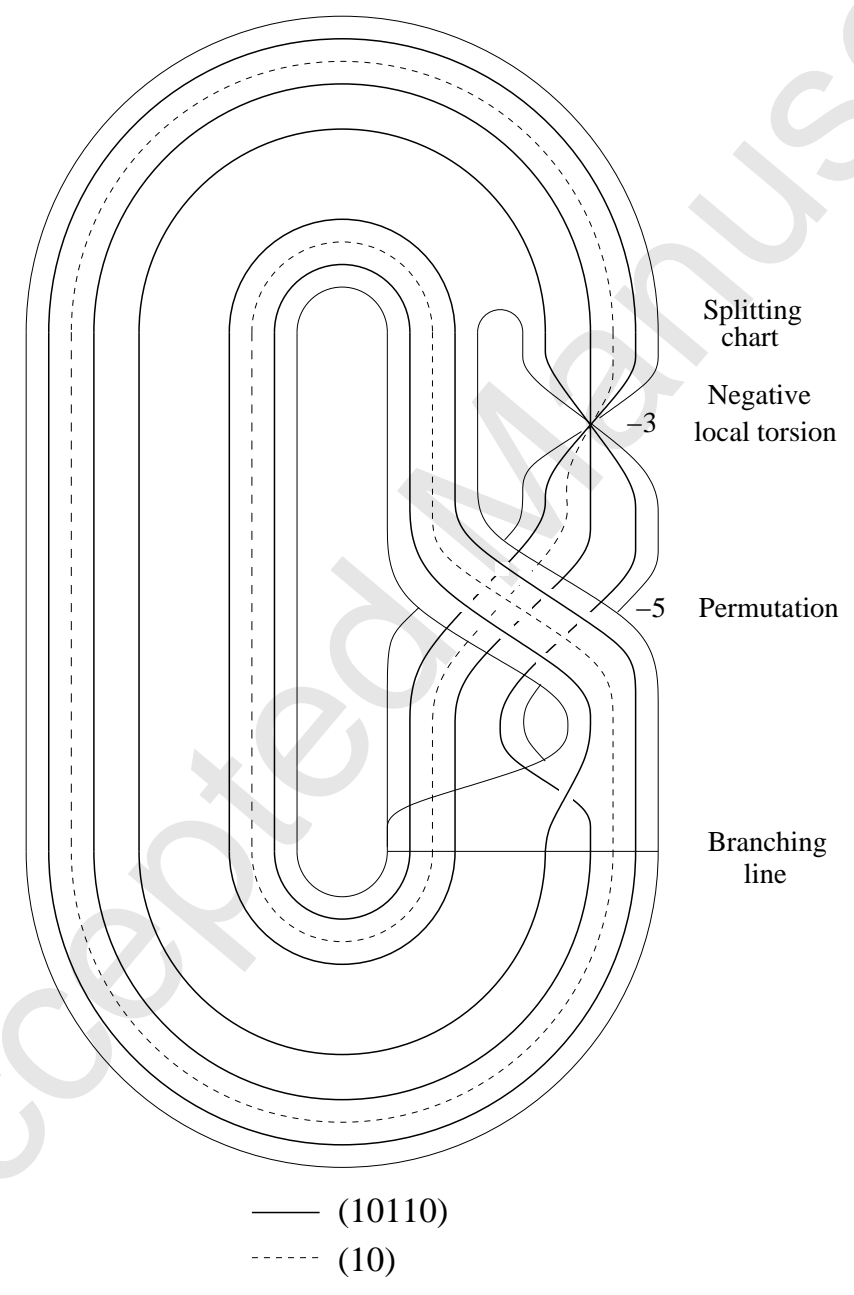

Figure 8: Template of the chaotic attractor shown in Fig. $5 \mathrm{~d}$ drawn with the period-2 orbit (10) and the period-5 orbit (10110). Eight negative crossings were counted on the template as in the regular plane projection shown in Fig. 7

We compute two two-dimensional bifurcation diagrams as shown in Figs. 9. The first one is spanned by the two time delays $\tau_{1}$ and $\tau_{2}$ (Fig. 9a). It clearly shown that for a given value of $\tau_{2}$, varying the delay $\tau_{1}$ with which tumor cells reduce the activity of immune cells does not affect too much the dynamics 
produced by system (4); in other terms, how fast tumor cells react to immune cells is not so relevant for tumor growth. Moreover, the inhibition rate of effector cells due to tumor cells does neither affect significantly the dynamics as evidenced by the bifurcation diagram shown in Fig. 10.
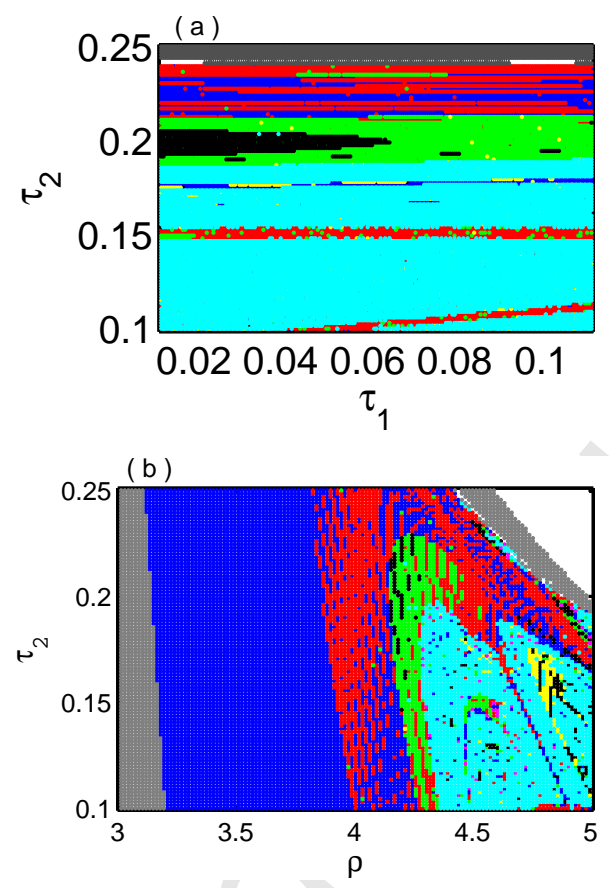

Figure 9: (Color online) Two-dimensional bifurcation diagrams of (a) $\tau_{1}$ vs. $\tau_{2}, \rho=4.5$ (b) $\rho$ vs. $\tau_{2}, \tau_{1}=0.012$ of system 44. Other parameter values as reported in Tab. 1 . Color legend as follows: Grey = stable singular point; blue = period-1 limit cycle; red = period-2 limit cycle; green = period-3 limit cycle; black = period-4 limit cycle; yellow = period-5 limit cycle; cyan $=$ period $\geq 6$ limit cycle or chaotic attractor; white $=$ unbounded state.

The second two-dimensional bifurcation diagram is spanned by the recruitment of effector cells by tumor cells $\rho$ and the second time delay $\tau_{2}$ (Fig. $9 \mathrm{~b}$ ). When $\tau_{2}$ is set to a given value, increasing $\rho$ develops the dynamics, that is, new unstable periodic orbits are created up to have a chaotic attractor (in the cyan domain). Depending on $\tau_{2}$ value, when $\rho$ is too much increased, reverse bifurcation can occur and some unstable periodic orbits are pruned. For a given value of the recruitment of effector cells by tumor cells $\rho$, increasing the delay $\tau_{2}$ induces a pruning of the population of unstable periodic orbits and the dynamics is "restricted" (not developed). This means that when effector cells are too long to react for killing tumor cells, the dynamics moves - in the parameter space - toward a stable three-cell singular point: tumor growth can thus occur more easily. From a clinical point of view, this would mean that increasing too much the delay $\tau_{2}$ with which the immune system kills tumor cells tends to reduce the resistance of the environment to tumor growth and therefore would induce a bad prognostic. This second bifurcation diagram, compared to the diagram shown in Fig. 9a, shows that the dynamics 


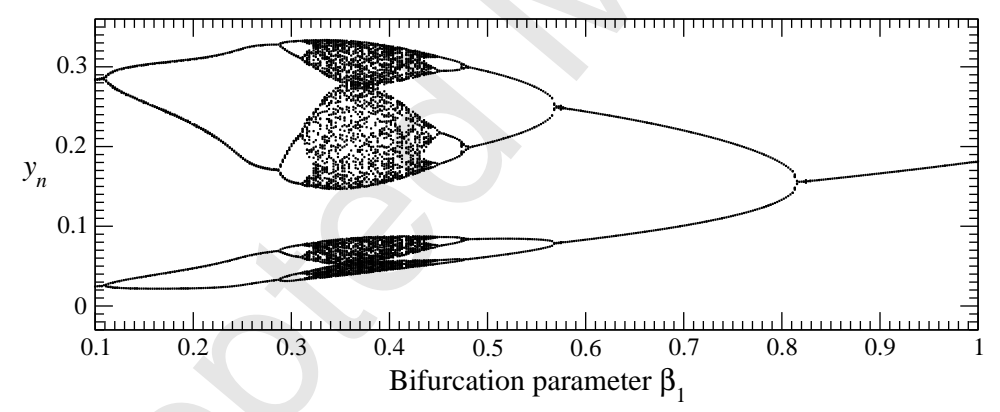

Figure 10: Bifurcation diagram versus $\beta_{1}$ of system (4). Other parameter values as reported in Tab. 1 . $\rho=4.5, \tau_{1}=0.012$ and $\tau_{2}=0.20$.

depends much more on delay $\tau_{2}$ and parameter $\rho$ than on delay $\tau_{1}$.

\section{Equivalence with a model without delay}

In order to identify parameter values that a system without delays would have for producing an attractor topologically equivalent to the attractor produced by system (4) with two delays, we first produced a multivariate time series made of the evolution of the three variables of the latter system (we used for this parameter values as used for Fig. 6). Then, a global modeling technique was applied to these three time series. The objective of a global modeling technique is to automatically produce a set of 

necessarily approximated — for a polynomial global model.

As a first step, the technique for selecting the polynomial structure returns the set of differential equation - without delays - as

$$
\left\{\begin{array}{l}
\dot{x}=a_{1} x+a_{2} x z \\
\dot{y}=b_{1} y+b_{2} y^{2}+b_{3} y z \\
\dot{z}=c_{1} z+c_{2} z^{2}+c_{3} x z+c_{4} y z
\end{array}\right.
$$

that is, with nearly the correct structure of system (4). The single difference being that term $a_{2} x z$ should be $a_{2} \frac{x z}{1+z}$. The numerical values of the coefficients provided by a chaotic attractor whose shape was close to the original one but the first-return map to a Poincaré section was slightly deformed compared to the original one shown in Fig. 6. Commonly, the development of chaotic attractor produced by a global model can be adjusted to the desired behavior by varying one of its parameter values. In spite of this, all our trials failed to reproduce correctly the target dynamics. This was only possible by replacing the term $x z$ by $\frac{x z}{1+z}$ in the first equation. Then, parameter $b_{1}$ was only slightly varied to produce the dynamical regime shown in Fig. 11. The global model without delays producing an attractor topologically equivalent to the original one is thus

$$
\left\{\begin{aligned}
\dot{x} & =-0.407 x+2.780 \frac{x z}{1+z} \\
\dot{y} & =0.533(1-0.9456 y)-1.497 y z \\
\dot{z} & =0.753(1-0.8486 z)-0.703 y z-2.323 x z
\end{aligned}\right.
$$

The topological properties of the resulting attractor were carefully compared with the original ones, and the values of parameter $\rho$ in the second equation was adjusted for having the same population of unstable periodic orbits embedded within the attractor (checked for orbits whose period is less than 9 as 


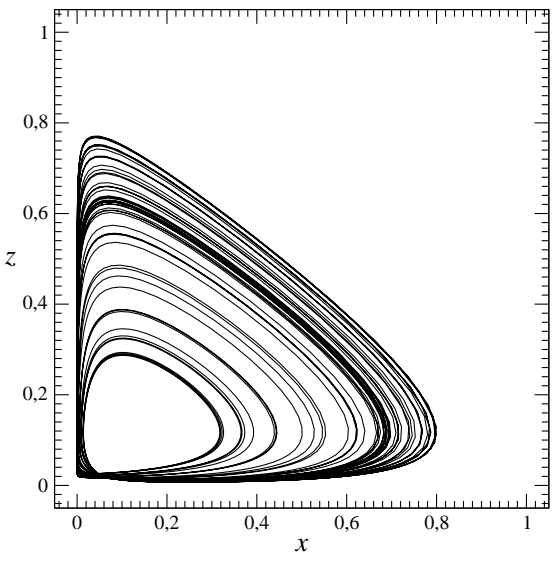

(a) State portrait

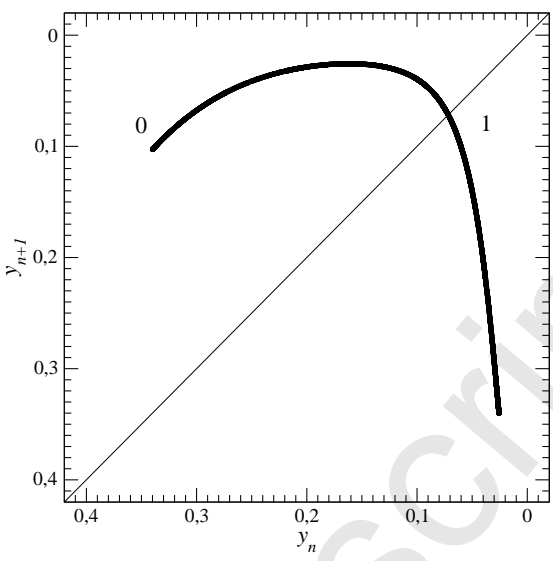

(b) First-return map

Figure 11: Chaotic attractor produced by the system with two delays and by the global model estimated from time series produced with that system.

reported in Table 22. The attractor is fully described by the template obtained for the original attractor (Fig. 8).

Table 2: Population of unstable periodic orbits embedded within the chaotic attractor produced by system (4) with two delays and by the global model 58 without delays. Only orbits whose period is less than 9 are reported.

\begin{tabular}{|c|c|c|c|c|c|}
\hline$\sigma$ & with delays & without & $\sigma$ & with delays & without \\
\hline (1) & • & $\bullet$ & $(10111111)$ & $\bullet$ & $\bullet$ \\
\hline (10) & & $\bullet$ & $(1011111)$ & $\bullet$ & $\bullet$ \\
\hline (1011) & $\bullet$ & $\bullet$ & (1011110) & • & $\bullet$ \\
\hline (10111010) & & $\bullet$ & (10111) & $\bullet$ & $\bullet$ \\
\hline (101110) & $\bullet$ & • & (10110) & - & $\bullet$ \\
\hline (101111) & $\bullet$ & $\bullet$ & $(1011010)$ & $\bullet$ & $\bullet$ \\
\hline (10111110) & • & $\bullet$ & $(1011011)$ & - & • \\
\hline
\end{tabular}

When parameter values are compared (see Table 1), it can be first noted that the growth rate $\rho$ of effector cells is smaller (2.780) than the original values (4.5) but this is most likely the result of the fact that the term $-\beta_{1} x z$ merged with term $\rho \frac{x z}{1+z}$ since $\beta_{1}=0$ in the global model (58), thus inducing necessarily a reduced value for $\rho$. When there is no delays, the decay rate of effector cells is smaller by about $20 \%$ than the original value. The other significantly different values are the tumor cell inactivation $\beta_{2}$ rate by effector cells (smaller by about $7 \%$ without delays). The delay $\tau_{2}$ with which the immune cells respond to the presence of tumor cells is therefore balanced by a stronger action of former cells on 
latter ones. The reduction of $\rho$ in the global model (58) is therefore also due to the presence of these two delays. Another consequence of the presence of the two delays is that it requires stronger host cells in their competition with tumor cells for nutrients and oxygen since, without delays, the inactivation rate $\gamma_{2}$ of tumor cells by host cells is reduced by about $30 \%$. Consequently, and not surprisingly, delays in the response of the immune system decrease the strength of the barriers provided by host and immune cells against tumor growth. With our global model, we showed that these delays do not induce dynamical regimes which are not observed when there is no delays.

\section{Discussion}

Investigating tumor growth taking into account the microenvironment received much attention although most of the studies were focussed on the immune system [74. The relevance of other stromal cells were only recently recognized [75, 76, 77, 78. These interactions are responsible for the differences between the doubling times observed in culture cancer cell lines and the doubling times of tumor observed in patients [79]. Moreover, treatments targeting host cells such as immunotherapy 33] and anti-angiogenic therapy [80, 81] showed significant anti-tumor effects in numerous solid tumors. Interactions between host and malignant cells in the tumor microenvironment could thus become the new key in future cancer therapy and new treatment strategy. Investigating models taking into account these interactions is therefore of a crucial interest not only for understanding tumor growth but also for designing new therapies. In this paper we investigated a model describing interactions between host, immune and tumor cells at the tissue level whose dynamics qualitatively correspond to various clinical features [18. Since some of these interactions can be clearly delayed in time, we introduced two time delays in i) the term describing how tumor cells inhibit the activity of immune cells and ii) the term corresponding to the inhibition of tumor cells by effector cells.

What is important to note here is that all the behaviors which were obtained with our delay differential model were already observed in the related model without delays $\left(\tau_{1}=\tau_{2}=0\right)$ as investigated in [18]. In particular the dormant cancer observed with large growth rate of host cells $(\alpha=1$ rather than 0.5 as used for Fig. 5d) was reproduced with nonzero delays. The delay value did not affect the fact that varying the tumor inhibition rate $\beta_{2}$ by the effector cells does not induce any obvious bifurcation. As suggested by our numerical simulations, introducing delays in the model does not induce any new dynamics compared to those produced by the non-delay system. By using a global modeling technique, we were able to obtain the parameter values required for producing a chaotic attractor with a system without delays and which is topologically equivalent to the attractor obtained with the system with two delays. Our ability to do that for a chaotic attractor - one of the most difficult regime to reproduce accurately - shows that any regimes produced with delays can be reproduced by a system without delays. The displacement in 
the parameter space show that larger the delays we introduced in the response of the immune system to the presence of tumor cells, stronger the proliferation of tumor cell is. To balance the effect of these delays, it is necessary to increase the growth rate of immune cells and the strength of host cells in their competition against tumor cells. Due to the additional complexity for investigating the solutions of systems with delays, we would recommend to not insert delays in cancer model until a specific action on the delay with which immune cells respond to tumor cells is specifically investigated.

\section{References}

[1] Schuch G, Kisker O, Atala A, and Soker S, Pancreatic tumor growth is regulated by the balance between positive and negative modulators of angiogenesis, Angiogenesis 5: 181-190, 2002.

[2] Laurent A, Nicco C, Chéreau C, Goulvestre C, Alexandre J, Alves A, Lévy E, Goldwasser F, Panis Y, Soubrane O, Weill B, Batteux F, Controlling tumor growth by modulating endogenous production of reactive oxygen species, Cancer Research 65: 948-956, 2005.

[3] Chew V, Toh HC, Abastado J-P, Immune microenvironment in tumor progression: Characteristics and challenges for therapy, J Oncol 2012: 608406, 2012.

[4] Norrby K, Metronomic chemotherapy and anti-angiogenesis: can upgraded pre-clinical assays improve clinical trials aimed at controlling tumor growth?, Acta Pathologica, Microbiologica et Immunologica Scandinavica 122: 565-579, 2014.

[5] Sun Y, Campisi J, Higano C, Beer TM, Porter P, Coleman I, True L, and Nelson PS, Treatmentinduced damage to the tumor microenvironment promotes prostate cancer therapy resistance through WNT16B, Nature Medicine 18: 1359-1368, 2012.

[6] Wheelock EF, Weinhold KJ, and Levich J, The tumor dormant state, Advances in Cancer Research 34: 107-140, 1981.

[7] Bissell MJ, and Hines WC, Why don't we get more cancer? A proposed role of the microenvironment in restraining cancer progression, Nature Medicine 17: 320-329, 2011.

[8] Eftimie R and Bramson JL, Interactions between the immune system and cancer: a brief review of non-spatial mathematical models, Bull. Math. Biol. 73: 2-32, 2011.

[9] d'Onofrio A, A general framework for modeling tumor-immune system competition and immunotherapy: Mathematical analysis and biomedical inferences, Physica D, 208: 220-235, 2005. 
[10] Kuznetsov VA, Makalkin IA, Taylor MA, and Perelson AS, Non-linear dynamics of immunogenic tumors: parameter estimation and global bifurcation analysis, Bull. Math. Biol. 56: 295-321, 1994.

[11] Kirschner D, and Panetta JC, Modeling the immunotherapy of tumor-immune interaction, J. Math. Biol. 37: 235-252, 1998.

[12] Chaplain MAJ, (eds.), Special issue on Mathematical models for the growth, development and treatment of tumors, Mathematical Models and Methods in Applied Science 9: 1999.

[13] Galach M, Dynamics of the tumor-tmmune system competition: The effect of the time delay, Int. J. Appl. Math. Comput. Sc. 13: 395-406, 2003.

[14] Pillis LG de, Radunskaya A, The dynamics of an optimally controlled tumor model: a case study, Math Comput Model 37: 1221-1244, 2003.

[15] de Pillis LG, Gu W, and Radunskaya AE, Mixed immunotherapy and chemotherapy of tumors: modeling, applications and biological interpretations, J. Theor. Biol. 238: 841-862, 2006.

[16] Reppas A, Alfonso JCL and Hatzikirou H, In silico tumor control induced via alternating immunostimulating and immunosuppressive phases, Virulence, 7: 174-186, 2016.

[17] Itik M, and Banks SP, Chaos in a three-dimensional cancer model, Int. J. Bifurcation Chaos 20: 71-79, 2010.

[18] Letellier C, Denis F, Aguirre LA, What can be learned from a chaotic cancer model?, J Theor Biol 322:7-16, 2013.

[19] Viger L, Denis F, Rosalie M and Letellier C, A cancer model for the angiogenic switch, J. Theor. Biol. 360: 21-33, 2014.

[20] Folkman J, Angiogenesis in cancer, vascular, rheumatoid and other disease, Nature Medicine 1: 27-31, 1995.

[21] Merlo LM, Pepper JW, Reid BJ, and Maley CC, Cancer as an evolutionary and ecological process, Nature Review in Cancer 6: 924-935, 2006.

[22] Malanchi I, Santamaria-Martinez A, Susanto E, Peng H, Lehr HA, Delaloye JF, and Huelsken J, Interactions between cancer stem cells and their niche govern metastatic colonization, Nature $\mathbf{4 8 1}$ : 85-89, 2012. 
[23] Mayer H, Zaenker KS, and an der Heiden U, A basic mathematical model of the immune response,

[24] Byrne HM, The effect of time delay on the dynamics of avascular tumor growth, Math. Biosc. 144: 83-117, 1997.

[25] Villasana M, and Radunskaya A, A delay differential equation model for tumor growth, J. Math. Biol. 47: 270-294, 2003.

[26] Yu C, and Wei J, Stability and bifurcation analysis in a basic model of the immune response with delays, Chaos, Solitons \& Fractals 41: 1223-1234, 2009.

[27] d'Onofrio A, and Gandolfi A, Tumour eradication by antiangiogenic therapy: analysis and extensions of the model by Hahnfeldt et al. (1999), Math. Biosci. 191: 159-184, 2004.

[28] Bi P, and Ruan S, Bifurcations in delay differential equations and applications to tumor and immune system interaction models, SIAM J. Appl. Dyn. Syst. 12: 1847-1888, 2013.

[29] Bi P, Ruan S, and Zhang X, Periodic and chaotic oscillations in a tumor and immune system interaction model with three delays, Chaos 24: 023101, 2014.

[30] Khajanchi S, and Banerjee S, Stability and bifurcation analysis of delay induced tumor-immune interaction model, Appl. Math. Comput. 248: 652-671, 2014.

[31] Garcia-Barros M, Paris F, Cordon-Cardo C, Lyden D, Rafii S, Haimovitz-Friedman A, Fuks Z, and Kolesnick R, Tumor response to radiotherapy regulated by endothelial cell apoptosis, Science 300: 1155-1159, 2003.

[32] Bochet L, Meulle A, Imbert S, Salles B, Valet P, and Muller C, Cancer-associated adipocytes promotes breast tumor radioresistance, Biochemical and Biophysical Research Communications. 411: 102-106, 2011.

[33] Topalian SL, Hodi FS, and Brahmer JR, Safety, activity, and immune correlates of anti-PD-1 antibody in cancer, N. Eng. J. Med. 28: 2443-2454, 2012.

[34] d'Onofrio A, Gatti F, Cerrai P and Freschi L, Delay-induced oscillatory dynamics of tumourimmune system interaction, Math. Comput. Modelling, 51: 572-591, 2010.

[35] Piotrowska MJ, An immune system-tumour interactions model with discrete time delay: model analysis and validation, Communications in Nonlinear Science and Numerical Simulation, 34: 185-198,2016. 
[36] Hale J, and Lunel SV, SV, Introduction to functional differential equations, Springer-Verlag: New York, 1993.

[37] Hale JK, and Sternberg N, Onset of chaos in differential delay equations, J. Comput. Phy. 77: 221-239, 1988.

[38] Wiggins S, Introduction to Applied Nonlinear Dynamical Systems and Chaos, Springer: New York, 1990.

[39] Brahmer JR, Tykodi SS, Chow LQ, Hwu WJ, Topalian SL, Hwu P, Drake CG, Camacho LH, Kauh J, Odunsi K, Pitot HC, Hamid O, Bhatia S, Martins R, Eaton K, Chen S, Salay TM, Alaparthy S, Grosso JF, Korman AJ, Parker SM, Agrawal S, Goldberg SM, Pardoll DM, Gupta A and Wigginton JM, Safety and activity of anti-PD-L1 antibody in patients with advanced cancer, N. Engl. J. Med. 366: 2455, 2012.

[40] Brahmer JR, Reckamp KL, Baas P, Crin L, Eberhardt WE, Poddubskaya E, Antonia S, Pluzanski A, Vokes EE, Holgado E, Waterhouse D, Ready N, Gainor J, Arén Frontera O, Havel L, Steins M, Garassino MC, Aerts JG, Domine M, Paz-Ares L, Reck M, Baudelet C, Harbison CT, Lestini B and Spigel DR, Nivolumab versus Docetaxel in advanced squamous-cell non-small-cell lung cancer, N. Engl. J. Med. 373: 123, 2015.

[41] Hatzikirou H, Alfonso JCL, Muehle S, Stern C, Weiss S and Meyer-Hermann M., Therapeutic potential of combinatorial anti-tumor treatments involving immuno- and vaso-modulatory interventions, J. R. Soc. Interface, 12: 20150439, 2015.

[42] Zeidler R, Reisbach G, Wollenberg B, Lang S, Chaubal S, Schmitt B, and Lindhofer H, Simultaneous activation of $\mathrm{T}$ cells and accessory cells by a new class of intact bispecific antibody results in efficient tumor cell killing, J. Immunol. 163: 1246-1252, 1999.

[43] Seruga B, Zhang H, Bernstein LJ, and Tannock IF, Cytokines and their relationship to the symptoms and outcome of cancer, Nature Reviews Cancer 8: 887-899, 2008.

[44] Mizoguchi AC, O'Shea H, Longo JJ, Loeffler DL, McVicar CM, and Ochoa DW, Alterations in signal transduction molecules in T lymphocytes from tumor-bearing mice,Science 258: 1795-1798, 1992.

[45] Drake CG, Jaffee E, and Pardoll DM, Mechanisms of immune evasion by tumors, Advances in Immunology. 90: 51-81, 2006.

[46] Mellman I, Coukos G, and Dranoff G, Cancer immunotherapy comes of age, Nature 480: 480-489, 2011. 
[47] Yang X, Chen L, and Chen J, Permanence and positive periodic solution for the single-species nonautonomous delay diffusive models, Computers $\&$ Mathematics with Applications. 32: 109$116,1996$.

[48] Diekmann O, van Giles S, and Lunel S, Delay Equations, Springer-Verlag: New York, 1995.

[49] Schatzman M, Numerical analysis: a mathematical introduction, Oxford University Press, 2002.

[50] Weigelt B, Ghajar CM, and Bissell MJ, The need for complex 3D culture models to unravel novel pathways and identify accurate biomarkers in breast cancer, Advanced Drug Delivery Reviews 69-70: 42-51, 2014.

[51] Klein G, Evolutionary aspects of cancer resistance, Seminars in Cancer Biology. 25: 10-14, 2014.

[52] Ruan SG, and Wei JJ, On the zeros of transcendental functions with applications to stability of delay differential equations with two delays, Dynamics of Continuous, Discrete and Impulsive Systems A 10: 863-874, 2003.

[53] Dieudonné J, Foundations of Modern Analysis, Academic Press: New York, 1960.

[54] Freedman HI, and Rao VSH, The trade-off between mutual interference and time lags in predatorprey systems, Bull. Math. Biol. 45: 991-1004, 1983.

[55] Hassard BD, Kazarinoff ND, and Wan YH, Theory and Application of Hopf Bifurcation, Chembridge University Press: Chembridge, 1981.

[56] Heun K, Neue methoden zur approximativen integration der differentialgleichungen einerunabhängigen Veränderlichen, Zeitschrift für angewandte Mathematik und Physik 45: 23-38, 1900.

[57] Cooke K, and van den Driessche P, On the zeros of some transcendental equations, Funkcialaj Ekvacioj 29: 77-90, 1986.

[58] Viger L, Denis F, Draghi C, Ménard T and Letellier C, Spatial avascular growth of tumor in a homogeneous environment, Journal of Theoretical Biology, 416: 99-112, 2016.

[59] Myrberg PJ, Sur l'itération des polynomes réels quadratiques, Journal de Mathématiques Pures et Appliquées 41: 339-351, 1962.

[60] Letellier C, Dutertre P, and Maheu B, Unstable periodic orbits and templates of the Rössler system: toward a systematic topological characterization, Chaos 5: 271-282, 1995.

[61] Rosalie R, and Letellier C, Systematic template extraction from chaotic attractors : I. Genus-one attractors with an inversion symmetry, J. Phys. A 46: 375101, 2013. 
[62] Gilmore R, and Lefranc M, The topology of chaos, Wiley, 2002.

[63] Tufillaro NB, Abbott T, and Reilly J, An experimental approach to nonlinear dynamics and chaos, Addison-Wesley: New York, 1992.

[64] Crutchfield JP and McNamara BS, Equations of motion from a data series, Complex Systems, 1: 417-452, 1987.

[65] Gouesbet G and Letellier C, Global vector field reconstruction by using a multivariate polynomial $L_{2}$-approximation on nets, Physical Review E, 49: 4955-4972, 1994.

[66] Aguirre LA and Letellier C, Modeling nonlinear dynamics and chaos: A review, Mathematical Problems in Engineering, 2009: 238960, 2009.

[67] Mangiarotti S, Coudret R,Drapeau L and Jarlan L, Polynomial search and global modeling: two algorithms for modeling chaos, Physical Review E, 86: 046205, 2012.

[68] Mangiarotti S, Drapeau L and Letellier C, Two chaotic global models for cereal crops cycles observed from satellite in northern Morocco, Chaos, 24: 023130, 2014.

[69] Mangiarotti S, Low dimensional chaotic models for the plague epidemic in Bombay (1896-1911), Chaos, Solitons \& Fractals, 81: 184-196, 2015.

[70] Aguirre LA, Billings SA, Dynamical effects of overparametrization in nonlinear models, Physica D, 80: 26-40 1995 .

[71] Aguirre LA, Freitas US, Letellier C and Maquet J, Structure-selection techniques applied to continuous-time nonlinear models, Physica D, 158: 1-18, 2001.

[72] Lainscsek C, Letellier C and Gorodnitsky I, Global modeling of the Rssler system from the zvariable, Physics Letters A, 314: 409-427, 2003.

[73] Lainscsek C, Letellier C and Schürrer C, Ansatz library for global modeling with a structure selection, Physical Review E, 64: 016206, 2001.

[74] Fridman WH, Dieu-Nosjean MC, Pagès F, Cremer I, Damotte D, Sautès-Fridman C, and Galon J, The immune microenvironment of human tumors: general significance and clinical impact, Cancer Microenvironment 6: 117-122, 2013.

[75] Li H, Fan X, and Houghton J, Tumor microenvironment: the role of the tumor stroma in cancer, J. Cell. Biochem. 101: 805-815, 2007. 
[76] Banerjee S, Khajanchi S, Chaudhury S, A mathematical model to elucidate brain tumor abrogation by immunotherapy with T11 target structure, PLoS ONE 10(5): e0123611, 2015.

[77] Mao Y, Keller ET, Garfield GH, Shen K, and Wang J, Stromal cells in tumor microenvironment and breast cancer, Cancer Metastasis Review 32: 303-315, 2013.

\section{AppendixA. Domain of existence of the limit cycle}

This appendix provides an additional support for estimating the delay values for which the limit cycle resulting from a Hopf bifurcation at the three-cell singular point $E^{*}$ persists. We started by estimating the delay window $\tau_{M}=\max \left\{\tau_{1}, \tau_{2}\right\}$ over which the periodic solution remains stable. Let us assume that system (4) is defined on $[-\tau,+\infty)$, the space of all continuous real-valued function defined in $S_{+}$ satisfying initial conditions (5) on $[-\tau, 0)$. First, we linearize system (4) around the three-cell singular point $E^{*}$, leading to

$$
\left\{\begin{array}{l}
\dot{x}=\frac{\rho z^{*}}{g+z *} x-\delta x-\beta_{1} z^{*} x\left(t-\tau_{M}\right)+\frac{g \rho x^{*}}{\left(g+z^{*}\right)^{2}} z-\beta_{1} x^{*} z\left(t-\tau_{M}\right) \\
\dot{y}=-\alpha y^{*} y-\gamma_{1} y^{*} z \\
\dot{z}=-\beta_{2} z^{*} x\left(t-\tau_{M}\right)-\gamma_{2} z^{*} y+\left(1-2 z^{*}-\gamma_{2} y^{*}\right) z-\beta_{2} x^{*} z\left(t-\tau_{M}\right) .
\end{array}\right.
$$


Applying a Laplace transformation on both sides of A.1, we have

$$
\left\{\begin{aligned}
\left(\eta+\delta-\frac{\rho z^{*}}{g+z *}+\beta_{1} z^{*} e^{-\eta \tau_{M}}\right) L_{x}(\eta)= & \frac{g \rho x^{*}}{\left(g+z^{*}\right)^{2}} L_{z}(\eta)-\beta_{1} z^{*} e^{-\eta \tau_{M}} K_{x}(\eta) \\
& -\beta_{1} x^{*} e^{-\eta \tau_{M}} L_{z}(\eta)-\beta_{1} x^{*} e^{-\eta \tau_{M}} K_{z}(\eta)+\bar{x}(0) \\
\left(\eta+\alpha y^{*}\right) L_{y}(\eta)= & -\gamma_{1} y^{*} L_{z}(\eta)+\bar{y}(0) \\
\left(\eta+1-2 z^{*}-\gamma_{2} y^{*}+\beta_{2} x^{*} e^{-\eta \tau_{M}}\right) L_{z}(\eta)= & =-\beta_{2} z^{*} e^{-\eta \tau_{M}} L_{x}(\eta)-\beta_{2} z^{*} e^{-\eta \tau_{M}} K_{x}(\eta) \\
& -\gamma_{2} z^{*} L_{y}(\eta)-\beta_{2} x^{*} e^{-\eta \tau_{M}} K_{z}(\eta)+\bar{z}(0),
\end{aligned}\right.
$$

with

$$
K_{x}(\eta)=\int_{-\tau_{M}}^{0} e^{-\eta t} x(t) d t
$$

and

$$
K_{z}(\eta)=\int_{-\tau_{M}}^{0} e^{-\eta t} z(t) d t
$$

and where $L_{x}(\eta), L_{y}(\eta)$ and $L_{z}(\eta)$ are Laplace transformations of $x(t), y(t)$ and $z(t)$, respectively. According to the theory developed by Freedman et al. [49] and using the classical Nyquist criterion, the singular point $E^{*}$ is stable when

$$
\operatorname{Re} B\left(i \xi_{0}\right)=0
$$

and

$$
\operatorname{Im} B\left(i \xi_{0}\right)>0
$$

with

$$
B(\eta)=\eta^{3}+a_{1} \eta^{2}+a_{2} \eta+a_{3}+e^{-\eta \tau_{M}}\left(b_{1} \eta^{2}+b_{2} \eta+b_{3}\right) .
$$

The minimal nonnegative root of equations A.3 and A.4 is $\xi_{0}>0$ with

$$
\left\{\begin{array}{l}
A_{1}=a_{1} \\
A_{2}=a_{2} \\
A_{3}=a_{3}
\end{array}\right.
$$

and

$$
\left\{\begin{array}{l}
B_{1}+C_{1}=b_{1} \\
B_{2}+C_{2}=b_{2} \\
B_{3}+C_{3}=b_{3} .
\end{array}\right.
$$

Rewriting equations A.3 and A.4 as

$$
-a_{1} \xi_{0}^{2}+a_{3}=-b_{2} \xi_{0} \sin \left(\xi_{0} \tau_{M}\right)-\left(b_{3}-b_{1} \xi_{0}^{2}\right) \cos \left(\xi_{0} \tau_{M}\right)
$$


and

$$
-\xi_{0}^{3}+a_{2} \xi_{0}>\left(b_{3}-b_{1} \xi_{0}^{2}\right) \sin \left(\xi_{0} \tau_{M}\right)-b_{2} \xi_{0} \cos \left(\xi_{0} \tau_{M}\right)
$$

provides sufficient conditions for the stability of the singular point $E^{*}$. To estimate the delay values we shall use the two conditions A.6 and A.7. Now our interest is to find the upper bound $\xi_{+}$for $\xi_{0}$ which is independent of $\tau_{M}$, the latter remaining to estimate. So condition A.7 holds for any value $0 \leq \xi \leq \xi_{+}$at $\xi=\xi_{0}$. Equation A.6 can thus be rewritten as

$$
a_{1} \xi_{0}^{2}=a_{3}+b_{2} \xi_{0} \sin \left(\xi_{0} \tau_{M}\right)+b_{3} \cos \left(\xi_{0} \tau_{M}\right)-b_{1} \xi_{0}^{2} \cos \left(\xi_{0} \tau_{M}\right) .
$$

For estimating the delay value, we maximize the right member of equation $\mathrm{A} .8$ where $\left|\cos \left(\xi_{0} \tau_{M}\right)\right| \leq 1$, and $\left|\sin \left(\xi_{0} \tau_{M}\right)\right| \leq 1$. Consequently, we have

$$
\left|a_{1}\right| \xi_{0}^{2} \leq\left|a_{3}\right|+\left|b_{2}\right| \xi_{0}+\left|b_{3}\right|+\left|b_{1}\right| \xi_{0}^{2}
$$

which can be expressed as

$$
\xi_{+} \leq \frac{1}{2\left(\left|a_{1}\right|-\left|b_{1}\right|\right)}\left[\left|b_{2}\right|+\sqrt{b_{2}^{2}+4\left(\left|a_{1}\right|-\left|b_{1}\right|\right)\left(\left|a_{3}\right|+\left|b_{3}\right|\right)}\right] .
$$

Hence it is obvious that $\xi_{0} \leq \xi_{+}$. Also, from condition (A.7), we have

$$
\xi_{0}^{2}<a_{2}+b_{2} \cos \left(\xi_{0} \tau_{M}\right)+b_{1} \xi_{0} \sin \left(\xi_{0} \tau_{M}\right)-\frac{b_{3} \sin \left(\xi_{0} \tau_{M}\right)}{\xi_{0}} .
$$

For $\tau_{M}=0$, this inequality has the form $\xi_{0}^{2}<a_{2}+b_{2}$, and from equation A.8,

$$
a_{1} \xi_{0}^{2}=a_{3}+b_{3}-b_{1} \xi_{0}^{2} \quad \Leftrightarrow \quad \xi_{0}^{2}=\frac{a_{3}+b_{3}}{a_{1}+b_{1}} .
$$

Therefore, we can assert that at $\tau_{M}=0$ the singular point $E^{*}$ is stable if

$$
\left(a_{3}+b_{3}\right)<\left(a_{1}+b_{1}\right)\left(a_{2}+b_{2}\right)
$$

holds. Now, for sufficiently small $\tau_{M}>0$, condition A.10 continues to hold.

Substituting equation A.8 into condition A.10, and rearranging the expression, we have

$$
\begin{gathered}
\left(b_{3}-b_{1} \xi_{0}^{2}-a_{1} b_{2}\right)\left[\cos \left(\xi_{0} \tau_{M}\right)-1\right]+\left[\left(b_{2}-a_{1} b_{1}\right) \xi_{0}+\frac{a_{1} b_{3}}{\xi_{0}}\right] \sin \left(\xi_{0} \tau_{M}\right) \\
<a_{1} a_{2}-a_{3}+a_{1} b_{2}-b_{3}+b_{1} \xi_{0}^{2} \\
\Leftrightarrow\left(b_{3}-b_{1} \xi_{0}^{2}-a_{1} b_{2}\right)\left[\cos \left(\xi_{0} \tau_{M}\right)-1\right]+\left[\left(b_{2}-a_{1} b_{1}\right) \xi_{0}+\frac{a_{1} b_{3}}{\xi_{0}}\right] \sin \left(\xi_{0} \tau_{M}\right) \\
<\left(a_{1}+b_{1}\right)\left(a_{2}+b_{2}\right)-\left(a_{3}+b_{3}\right) .
\end{gathered}
$$

Using the bounds, we obtain

$$
\begin{aligned}
\left(b_{3}-b_{1} \xi_{0}^{2}-a_{1} b_{2}\right)\left[\cos \left(\xi_{0} \tau_{M}\right)-1\right]= & 2\left(b_{1} \xi_{0}^{2}+a_{1} b_{2}-b_{3}\right) \sin ^{2}\left(\frac{\xi_{0} \tau_{M}}{2}\right) \\
& \leq \frac{1}{2} \xi_{+}^{2}\left|\left(b_{1} \xi_{+}^{2}+a_{1} b_{2}-b_{3}\right)\right| \tau_{M}^{2}
\end{aligned}
$$


and

$$
\left[\left(b_{2}-a_{1} b_{1}\right) \xi_{0}+\frac{a_{1} b_{3}}{\xi_{0}}\right] \sin \left(\xi_{0} \tau_{M}\right) \leq\left[\left|b_{2}-a_{1} b_{1}\right| \xi_{+}^{2}+\left|a_{1}\right| \cdot\left|b_{3}\right|\right] \tau_{M}
$$

From condition A.11 we obtain that $D_{1} \tau_{M}^{2}+D_{2} \tau_{M} \leq D_{3}$ with

$$
\left\{\begin{array}{l}
D_{1}=\frac{1}{2}\left|b_{1} \xi_{+}^{2}+a_{1} b_{2}-b_{3}\right| \xi_{+}^{2} \\
D_{2}=\left[\left|b_{2}-a_{1} b_{1}\right| \xi_{+}^{2}+\left|a_{1}\right| \cdot\left|b_{3}\right|\right] \\
D_{3}=\left(a_{1}+b_{1}\right)\left(a_{2}+b_{2}\right)-\left(a_{3}+b_{3}\right) .
\end{array}\right.
$$

Now it follows that

$$
\tau_{M}^{+}=\frac{1}{2 D_{1}}\left[-D_{2}+\sqrt{D_{2}^{2}+4 D_{1} D_{3}}\right]
$$

for $0 \leq \tau_{M} \leq \tau_{M}^{+}$and, consequently, the Nyquist criterion holds: the maximum delay value $\tau_{M}^{+}$ensures the existence of the limit cycle. 